\title{
HAK/KUP/KT family potassium transporter genes are involved in potassium deficiency and stress responses in tea plants (Camellia sinensis L.): expression and functional analysis
}

Tianyuan Yang ${ }^{\dagger}$, Xin Lư ${ }^{\dagger}$ Yan Wang ${ }^{\dagger}$, Yunxia Xie, Jingzhen Ma, Xunmin Cheng, Enhua Xia, Xiaochun Wan ${ }^{*}$ and Zhaoliang Zhang ${ }^{*}$

\begin{abstract}
Background: Tea plant is one of the most important non-alcoholic beverage crops worldwide. While potassium $\left(\mathrm{K}^{+}\right)$is an essential macronutrient and greatly affects the growth and development of plants, the molecular mechanism underlying $\mathrm{K}^{+}$uptake and transport in tea plant root, especially under limited- $\mathrm{K}^{+}$conditions, is still poorly understood. In plants, HAK/KUP/KT family members play a crucial role in $\mathrm{K}^{+}$acquisition and translocation, growth and development, and response to stresses. Nevertheless, the biological functions of these genes in tea plant are still in mystery, especially their roles in $\mathrm{K}^{+}$uptake and stress responses.
\end{abstract}

Results: In this study, a total of 21 non-redundant HAK/KUP/KT genes (designated as CsHAKs) were identified in tea plant. Phylogenetic and structural analysis classified the CsHAKs into four clusters (I, II, III, IV), containing 4, 8, 4 and 5 genes, respectively. Three major categories of cis-acting elements were found in the promoter regions of CsHAKs. Tissue-specific expression analysis indicated extremely low expression levels in various tissues of cluster I CsHAKs with the exception of a high root expression of CSHAK4 and CSHAK5, a constitutive expression of clusters II and III CsHAKs, and a moderate cluster IV CSHAKs expression. Remarkably, the transcript levels of CsHAKs in roots were significantly induced or suppressed after exposure to $\mathrm{K}^{+}$deficiency, salt and drought stresses, and phytohormones treatments. Also notably, CSHAK7 was highly expressed in all tissues and was further induced under various stress conditions. Therefore, functional characterization of CSHAK7 was performed, and the results demostrated that CsHAK7 locates on plasma membrane and plays a key role in $\mathrm{K}^{+}$transport in yeast. Taken together, the results provide promising candidate CsHAKs for further functional studies and contribute to the molecular breeding for new tea plants varieties with highly efficient utilization of $\mathrm{K}^{+}$.

Conclusion: This study demonstrated the first genome-wide analysis of CSHAK family genes of tea plant and provides a foundation for understanding the classification and functions of the CsHAKs in tea plants.

Keywords: Camellia sinensis, HAK/KUP/KT family, K+ acquisition, Stress responses, Gene expression profiles

\footnotetext{
*Correspondence: xcwan@ahau.edu.cn; zhlzhang@ahau.edu.cn

${ }^{\dagger}$ Tianyuan Yang, Xin Lu and Yan Wang contributed equally to this work. State Key Laboratory of Tea Plant Biology and Utilization, Anhui Agricultural University, Hefei 230036, Anhui, China
}

C C The Author(s). 2020 Open Access This article is licensed under a Creative Commons Attribution 4.0 International License, which permits use, sharing, adaptation, distribution and reproduction in any medium or format, as long as you give appropriate credit to the original author(s) and the source, provide a link to the Creative Commons licence, and indicate if changes were made. The images or other third party material in this article are included in the article's Creative Commons licence, unless indicated otherwise in a credit line to the material. If material is not included in the article's Creative Commons licence and your intended use is not permitted by statutory regulation or exceeds the permitted use, you will need to obtain permission directly from the copyright holder. To view a copy of this licence, visit http://creativecommons.org/licenses/by/4.0/ The Creative Commons Public Domain Dedication waiver (http://creativecommons.org/publicdomain/zero/1.0/) applies to the data made available in this article, unless otherwise stated in a credit line to the data. 


\section{Background}

Tea (the beverage) is made of bud and young leaves of tea plants (Camellia sinensis). Organic and inorganic components in young shoots determine the quality of the tea drink. Among these substances, catechins, theanine, and caffeine are the most important characteristic secondary metabolites in tea bud and leaf, which endow the tea with a rich taste and many health benefits [1-3]. Generally, the composition of the tea bud and young leaf is greatly influenced by many factors, such as the tea plant cultivar, nutrition status and environmental factors [4-7]. Out of these factors, mineral nutrition, especially nutrition of potassium, considerably affects the growth and development of tea plants [8]. For example, Ruan et al. (1998) reported that $\mathrm{K}^{+}$fertilizer application increased the contents of free amino acids, caffeine and polyphenols in leaves of the various types of the tea plant. Additionally, exogenous $\mathrm{K}^{+}$application or maintaining $\mathrm{K}^{+}$accumulation in mesophyll cells appeared to mitigate remarkably the tea plant drought stress [9].

$\mathrm{K}^{+}$is the most abundant cation in plant cells, accounting for 2 to $10 \%$ of plant dry matter. It is involved in many physiological processes including transmembrane transport, enzyme activation, anion neutralization, photosynthesis, osmoregulation, and stomatal movement $[10,11]$, thereby regulates plant growth, development and stress responses. Tea plants are preferably grown in tropical and subtropical regions where soil is usually highly leachy and acidic, and consequently, $\mathrm{K}^{+}$deficient [12]. Inadequate application of $\mathrm{K}$ fertilizer often imposes many adverse impacts on tea plant, especially reduces photosynthetic efficiency and enzyme activity, and therefore causes a great loss of quality and yield of tea [13].

Due to the combinational effects of chemical fixation, root absorption, and loss by leaching, the $\mathrm{K}^{+}$concentration in rhizosphere varies and rarely exceeds $1 \mathrm{mM}$ [14]. Faced with the requirement of approximately $100 \mathrm{mM}$ $\mathrm{K}^{+}$concentration in plant cell cytoplasm for adequate metabolic function [10, 15], plants have evolved multiple $\mathrm{K}^{+}$uptake systems in adaptation to this imbalance and variability [16-18]. Among these, HAK/KUP/KT (highaffinity $\mathrm{K}^{+}$transporter / $\mathrm{K}$ uptake permease / $\mathrm{K}^{+}$transporter) constitutes the largest gene family of plant $\mathrm{K}^{+}$ transporters which mediate $\mathrm{K}^{+}$uptake at micromolar range [19, 20].

In the past decades, a series of plant $H A K / K U P / K T$ genes were identified and their multiple physiological roles were characterized in many plant species [21, 22]. For instance, a total of $13 \mathrm{HAK/KUP/KT}$ family members have been identified in the model plant Arabidopsis (A. thaliana) and 27 OsHAKs in rice (Oryza sativa). To date, with the release of genomes, HAK/KUP/KT family members have also been identified and characterized in other plant species, such as maize (Zea mays) [23], poplar (Populus trichocarpa) [24], tomato (Solanum lycopersicum) [25], soybean (Glycine max) [26] and pear (Pyrus bretschneideri) [27].

$H A K / K U P / K T s$ express in different plant tissues, such as root [28], leaf [29], seed [30], and fruit [31]. Their expression is also highly regulated by $\mathrm{K}^{+}$starvation [21], abiotic stresses including salt stress and drought [3234], and phytohormones, such as, ABA (abscisic acid) [29], ethylene [35, 36], cytokinin [37] and NAA (1naphthylacetic acid) [38]. These HAK/KUP/KT proteins demonstrated a great diversity of subcellular localization, such as plasma membrane [30, 32, 33], tonoplast [39], and endoplasmic reticulum [40]. Generally, they are shown to have $\mathrm{K}^{+}$uptake activity by conferring the growth of $\mathrm{K}^{+}$-uptake-deficient bacteria or yeast mutants when they express in these mutants [28, 39, 41, 42]. Plant HAK/KUP/KT proteins typically contain 10-15 transmembrane (TM) segments and include a conserved K transport domain (GVVYGDLGTSPLY) [42-44]. They were generally classified into four clusters (I-IV) based on phylogenetic analysis $[38,39]$.

Expression patterns and physiological roles of some $\mathrm{HAK} / \mathrm{KUP} / \mathrm{KTs}$ from cluster I were characterized in both Arabidopsis and rice [17, 18, 20]. For example, the transcript level of AtHAK5 was induced in root under $\mathrm{K}^{+}$-limitation conditions, while it was greatly downregulated by high salinity [45]. It was further demonstrated that AtHAK5 played a key role in high-affinity $\mathrm{K}^{+}$uptake under low $\mathrm{K}^{+}$concentration even below $10 \mu \mathrm{M}$ [46, 47]. In rice, OsHAK1, OsHAK5 and $O S H A K 21$ were greatly induced in roots exposing to $\mathrm{K}^{+}$ deficiency and salt stress, and were shown to be involved in the maintenance of $\mathrm{K}^{+}$homeostasis and salt tolerance [30, 32, 33]. Recently, Yang et al. (2020) showed that OsHAK5 is not only critical in $\mathrm{K}^{+}$uptake, but also alters rice architecture by regulating ATP-dependent auxin transport $[30,48]$ OsHAK1 was recently shown to mediate $\mathrm{K}^{+}$uptake under both low and high $\mathrm{K}^{+}$concentrations and positively regulate drought stress response [34].

HAK/KT/KUPs from cluster II are proposed to mainly function in plant growth and development. In Arabidopsis, AtKUP4/TRH1 (Tiny Root Hairs 1) functions in maintaining polar localization of AtPIN1 in root apex and establishing appropriate auxin gradients, which in turn promote root gravitropism response and root hair formation [40, 49, 50]. Knockout of AtKUP2/SHY3 (Short Hypocotyl 3) also caused developmental defects in shoots [51]. Notably, a triple null mutant atkup2/ 6/8 showed promoted plant growth and impaired abscisic acid (ABA) responses in guard cells and lateral root cells [29].

Up to date, the information about the functions of $H A K / K U P / K T$ genes from cluster III and IV is scarce 
[18, 20, 22]. Notably, however, AtHAK7 was shown to be involved in $\mathrm{K}^{+}$loading into xylem, and, consequently, in $\mathrm{K}^{+}$transport from root to shoot, especially under $\mathrm{K}^{+}$-deficiency [52].

Due to the difficulty in genetic transformation and slow growth of tea plants, much fewer genes were identified and characterized in comparison to model plants. However, a rapid progress in tea biotechnology and functional genomics was achieved recently [53-55]. For example, Alagarsamy et al. (2020) reported that they firstly established an optimized method of Agrobacterium-mediated transgenic hairy root induction of $C$. sinensis. With this method, functional characterization of the "in root" genes could be carried out in vivo in the tea plant [56]. More recently, a native C. sinensis Tannase gene (CsTA) was identified for the first time in plant kingdom [57]. The transcriptional and metabolic analyses revealed that the expression of CsTA negatively regulated the accumulation of galloylated catechins [57]. Moreover, Dong et al.(2020) showed that six amino acid permeases in tea plant exhibited theanine transport activity, and suggested a role for CsAPP1 in transporting theanine from roots toward new shoots [58]. Such progress related to tea plant biotechnology should contribute to a better understanding of the tea plant biological characteristics.

The study of the molecular underpins of the tea plant $\mathrm{K}^{+}$nutrition is also bound to benefit from the biotechnological progress. Although $\mathrm{K}^{+}$is one of the most essential macronutrients, its uptake and transport has not been studied in molecular level in the tea plants. HAK/ KUP/KT family has not been systematically identified in tea plant, either [55]. With the release of the tea plant genomes $[59,60]$, we currently have an opportunity to systemically identify HAK/KUP/KT family and investigate their physiological roles. In this study, 21 nonredundant CsHAKs were identified in the tea plant. Subsequently, gene structures, phylogenetic relationships, tissue-specific expression, and expression in responses to $\mathrm{K}^{+}$deficiency and various stresses were investigated. To further reveal the role of HAK/KUP/ KTs in the tea plant, the subcellular localization and functional characterization of CsHAK7 in $\mathrm{K}^{+}$acquisition were also performed. Overall, our results provide a foundation for further functional characterization of HAK/ $\mathrm{KUP} / \mathrm{KTs}$ in the tea plant and contribute to a better understanding of the molecular basis of CsHAKs' responses to $\mathrm{K}^{+}$deficiency and various stresses.

\section{Results}

Identification and sequence analysis of CsHAKs in the tea plant

To identify the complete set of CsHAK family members in the tea plant genome, the sequences of 13 Arabidopsis and 27 rice $\mathrm{HAK} / \mathrm{KUP} / \mathrm{KT}$ proteins were used as queries to screen the local tea plant genome database. Then, the HMM profile of the HAK domain (K_trans; PF02705) was performed to conduct a global search of the tea plants genome [60]. Next, both Pfam database and InterProScan database were used to further confirm the presence of the conserved HAK domain. A total of 21 non-redundant $C s H A K s$ were identified in the tea plant genome (Table 1). Due to the different number of CsHAKs between tea plant and Arabidopsis, therefore, we named some of these CsHAKs based on their homology with those of Arabidopsis, and the reminding are named freely. The detailed information on the CsHAKs (including gene name, sequence ID, protein length, molecular weight, theoretical isoelectric point, predicted subcellular localization) has been listed in Table 1. The predicted CsHAK proteins had considerably different amino acid lengths and molecular weights. Their lengths ranged from 691 to 947 amino acid residues. The values of their theoretical isoelectric points varied from 5.36 to 9.28. CsHAK17 had the lowest molecular weight (77.28 $\mathrm{kDa})$ and CsHAK14 had the highest $(105.26 \mathrm{kDa})$. Based on the prediction by TMME2.0 web server, the number of transmembrane segments (TMS) of CsHAKs ranged from 10 to 14 (Fig. S1). The majority of CsHAKs were predicted to localize to the plasma membrane, with several of them probably in the cytoplasm and chloroplast (Table 1).

\section{Phylogenetic analysis of CsHAKs}

To reveal the evolutionary relationship and functional divergence of the CsHAK family members, the full length of 111 HAK proteins, including 21 from tea plants, 13 from Arabidopsis, 27 from rice, 31 from poplar and 19 from grape, were used to construct a phylogenetic tree (Fig. 1). These HAK/KUP/KT proteins were classified into four clusters, with 4, 8, 4, 5 members, in clusters I, II, III and IV, respectively (Fig. 1). Clusters II and IV members were the most abundant in tea plants, comprising $61.90 \%$ of all CsHAKs. Importantly, these proteins, including CsHAK4, 5, 9 and 20, were distributed in cluster I together with the already-characterized AtHAK5 [46], OsHAK1 [32, 34] and OsHAK5 [30, 62], suggesting that they also have a crucial role in the tea plant $\mathrm{K}^{+}$uptake from a low- $\mathrm{K}^{+}$soil. Among the cluster II members, eight of the CsHAKs (CsHAK1, 2, 3, 6, 8, $13,17,21)$ were found together with AtKUP2 [51] and AtKUP4 [40, 49, 50], implying that they are likely involved in developmental processes in the tea plant. Notably, among members of cluster III, CsHAK7 shared the almost highest sequence identity (77.9\%) with AtKUP7 [52], suggesting a role for CsHAK7 in $\mathrm{K}^{+}$acquisition and translocation in the tea plant root in the presence of a low $\mathrm{K}^{+}$concentration. 
Table 1 Information of CsHAKs identified in tea plants

\begin{tabular}{|c|c|c|c|c|c|c|c|c|c|c|c|c|c|}
\hline \multirow[t]{2}{*}{ Name } & \multirow{2}{*}{$\begin{array}{l}\text { Sequence } \\
\text { ID }\end{array}$} & \multicolumn{12}{|l|}{ Protein } \\
\hline & & $\begin{array}{l}\text { Length } \\
\text { (aa) }\end{array}$ & $\mathrm{pl}$ & $\begin{array}{l}\mathrm{MW} \\
(\mathrm{KDa})\end{array}$ & $\begin{array}{l}\text { Subcellular } \\
\text { localization }\end{array}$ & $B$ & $\mathrm{FL}$ & FR & L1 & L2 & L3 & $R$ & $\mathrm{~S}$ \\
\hline$\overline{\text { CSHAK1 }}$ & TEA008833.1 & 714 & 8.10 & 80.03 & Plasma membrane & 110.9029 & 42.0600 & 11.2404 & 44.7742 & 3.8711 & 0.6120 & 14.1058 & 42.7326 \\
\hline CsHAK2 & TEA022010.1 & 802 & 8.48 & 89.68 & Cytoplasm & 27.9207 & 64.8636 & 21.2298 & 18.7921 & 1.9196 & 1.5295 & 3.1587 & 21.1625 \\
\hline CsHAK3 & TEA005066.1 & 787 & 8.64 & 87.40 & Plasma membrane & 51.2697 & 45.7785 & 59.9698 & 91.1863 & 85.0903 & 120.2709 & 68.3359 & 83.5590 \\
\hline CsHAK4 & TEA003872.1 & 752 & 7.25 & 84.26 & Plasma membrane & 0.2042 & 0.1092 & 0.3867 & 0.1139 & 0.0656 & 0.0222 & 0.7034 & 0.1293 \\
\hline CsHAK5 & TEA003873.1 & 809 & 8.47 & 91.38 & Plasma membrane & 0.0072 & 0.3697 & 0.0249 & 0.0075 & 0.0000 & 0.0100 & 18.7464 & 0.0085 \\
\hline CSHAK6 & TEA011201.1 & 767 & 8.60 & 86.69 & Cytoplasm & 19.8532 & 17.0587 & 50.9855 & 40.9706 & 16.1786 & 73.3125 & 9.4973 & 31.0653 \\
\hline CsHAK7 & TEA003094.1 & 908 & 5.74 & 101.11 & Plasma membrane & 11.5103 & 10.8720 & 13.0813 & 9.8839 & 13.4190 & 12.8203 & 10.8639 & 16.6949 \\
\hline CsHAK8 & TEA007724.1 & 805 & 7.32 & 90.78 & Plasma membrane & 10.3956 & 9.6716 & 18.6232 & 17.7413 & 14.0597 & 18.1328 & 7.5946 & 20.9987 \\
\hline CsHAK9 & TEA003884.1 & 736 & 9.28 & 82.90 & Plasma membrane & 0.1850 & 0.1677 & 0.0461 & 0.1167 & 0.1727 & 0.1268 & 0.1135 & 0.0378 \\
\hline CsHAK10 & TEA021417.1 & 855 & 7.32 & 95.86 & Plasma membrane & 47.0292 & 49.8357 & 25.9564 & 44.7135 & 39.1111 & 30.2252 & 70.2434 & 36.9693 \\
\hline SHAK11 & TEA006987.1 & 732 & 8.67 & 80.32 & Plasma membrane & 1.8167 & 1.9706 & 1.4882 & 8318 & 4.0013 & 3.7068 & 2.6004 & 3.9919 \\
\hline CsHAK12 & TEA032656.1 & 709 & 5.52 & 79.24 & chloroplast & 15.6754 & 9.6164 & 17.9050 & 13.8896 & 20.9087 & 10.2305 & 14.5307 & 20.7014 \\
\hline CsHAK13 & TEA023021.1 & 734 & 8.81 & 81.51 & Plasma membrane & 0.0645 & 41.9444 & 1.4596 & 0.1589 & 4.9184 & 0.8987 & 0.3129 & 2.4945 \\
\hline CsHAK14 & TEA011420.1 & 947 & 8.93 & 105.26 & Plasma membrane & 0.6758 & 1.5437 & 0.1622 & 0.4367 & 0.4758 & 0.4585 & 0.1869 & 12.1504 \\
\hline CsHAK15 & TEA012915.1 & 710 & 8.95 & 79.08 & Plasma membrane & 1.0015 & 2.3787 & 1.4008 & 0.5114 & 3.1257 & 0.4787 & 0.5389 & 10.8703 \\
\hline CsHAK16 & TEA016241.1 & 703 & 8.33 & 78.77 & Plasma membrane & 0.0085 & 67.3102 & 0.0388 & 0.0000 & 0.0000 & 0.0356 & 0.2088 & 0.0299 \\
\hline CsHAK17 & TEA018688.1 & 691 & 7.57 & 77.28 & Plasma membrane & 5.3419 & 19.1077 & 5.2523 & 7.2408 & 7.3490 & 21.1981 & 28.6248 & 14.8871 \\
\hline CsHAK18 & TEA023299.1 & 841 & 5.36 & 93.86 & Plasma membrane & 6.2981 & 20.2273 & 4.1755 & 5.4650 & 8.4885 & 10.9642 & 7.6997 & 8.8804 \\
\hline CsHAK19 & TEA025917.1 & 780 & 8.57 & 86.17 & Plasma membrane & 0.1056 & 0.2360 & 0.0605 & 0.0156 & 0.0810 & 0.0634 & 4.5690 & 0.1330 \\
\hline CsHAK20 & TEA031103.1 & 702 & 8.43 & 79.09 & Plasma membrane & 0.0254 & 0.0294 & 0.0000 & 0.0439 & 0.0000 & 0.0000 & 0.0000 & 0.0000 \\
\hline CsHAK21 & TEA032638.1 & 789 & 7.55 & 87.77 & Plasma membrane & 3.9471 & 4.2648 & 9.7922 & 2.2762 & 1.2882 & 25.0887 & 3.5484 & 3.3271 \\
\hline
\end{tabular}

\section{Gene structure and conserved motifs of CsHAKs}

To gain insights into the evolutionary relationships and structural features, the exon/intron structures of CsHAKs and conserved motifs in CsHAKs were investigated. CsHAK proteins in cluster I-IV were listed in order based on the phylogenetic analysis (Fig. 1 and Fig. 2a). The gene structure of CsHAKs was constructed by comparing the CDS and genomic sequences using an online gene structure display server (GSDS) 2.0 program. Most of members of CsHAKs possessed 6 to 10 exons and 5 to 9 introns except for CsHAK14 (15 exons /14 introns) (Fig. 2a). As expected, most of CsHAKs belonging to the same cluster also displayed similar distribution patterns of exon/intron in terms of exon length and intron number (Fig. 2a). This was in agreement with the results reported in rice [38] and wheat [63].

To investigate the distribution of conserved motifs in CsHAKs, a total of 20 conserved motifs were identified by the MEME program and were designated as motifs 1-20. The highly conserved motif containing a special $\mathrm{K}^{+}$transport domain (GVVYGDLGTSPLY) was emphasized with motif logo (motif 8) (Fig. 2b), and it existed in all CsHAK proteins (Fig. 2b; Table S4). Generally, the 20 motifs were almost evenly distributed in CsHAK proteins. Motifs 1, 2, 4, 5, 7 and 8 possessing feature domain of $\mathrm{K}^{+}$transporter (Fig. 2b, Table S3) were observed in all CsHAK proteins. Together, CsHAK proteins belonging to the same cluster exhibited a similar motif distribution, which was consistent with the results of gene structure analysis.

\section{Analysis of cis-elements in the CsHAKs promoters}

To understand the transcriptional regulation and function of CsHAKs, the promoter regions (2000 bp upstream of the transcription start site) were used to identify cis-elements using the PlantCARE database (Fig. 3, Fig. S2, Table S5). A total of twenty-three types of cis-acting elements were identified. These elements randomly distributed in the promoter regions of CsHAKs and were predicted to participate in plant growth and development responses, stress and phytohormone responses. Among the cis-acting elements related to plant growth, eight endosperm expression regulatory elements and eight zein metabolism regulation element were 


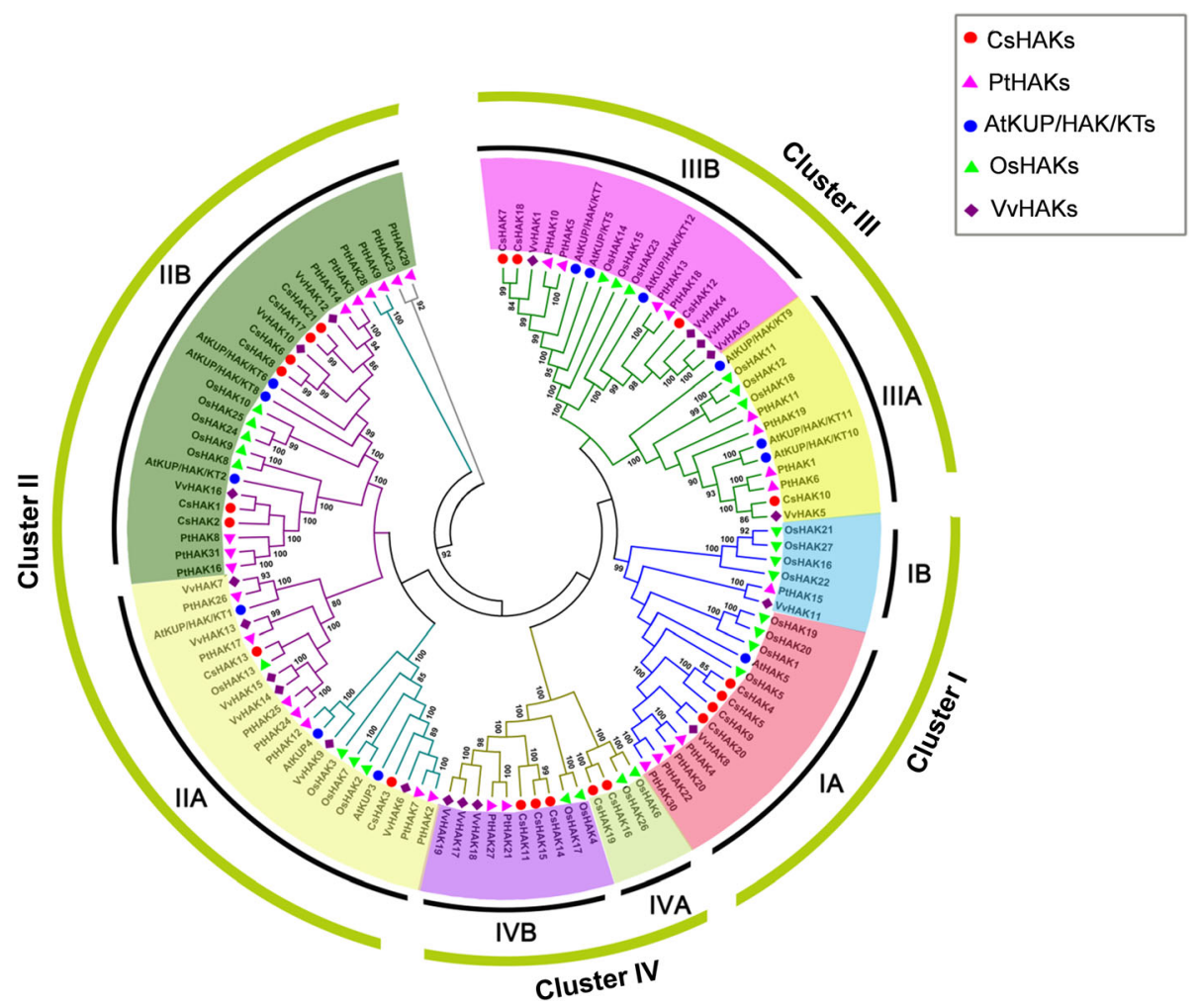

Fig. 1 Phylogenetic tree of KT/HAK/KUP family proteins among tea plant, poplar, rice, grape and Arabidopsis. The proteins belonging to each of the five species are represented by different colors and shapes. The KT/HAK/KUP family proteins were classified into four clusters (I, II, III, IV). Every cluster further were divided into two subgroups including cluster IA and IB, Cluster IIA and IIB, Cluster IIIA and IIIB, Cluster IVA and IVB, based on their sites in a same branch. The gene ID of tea plant, poplar, rice, grape and Arabidopsis KUP/HAK/KT family proteins are listed in Table S2. CS, Camellia sinensis; Pt, Populus trichocarpa; At, Arabidopsis thaliana; Os, Oryza sativa .Vv, Vitis vinifera

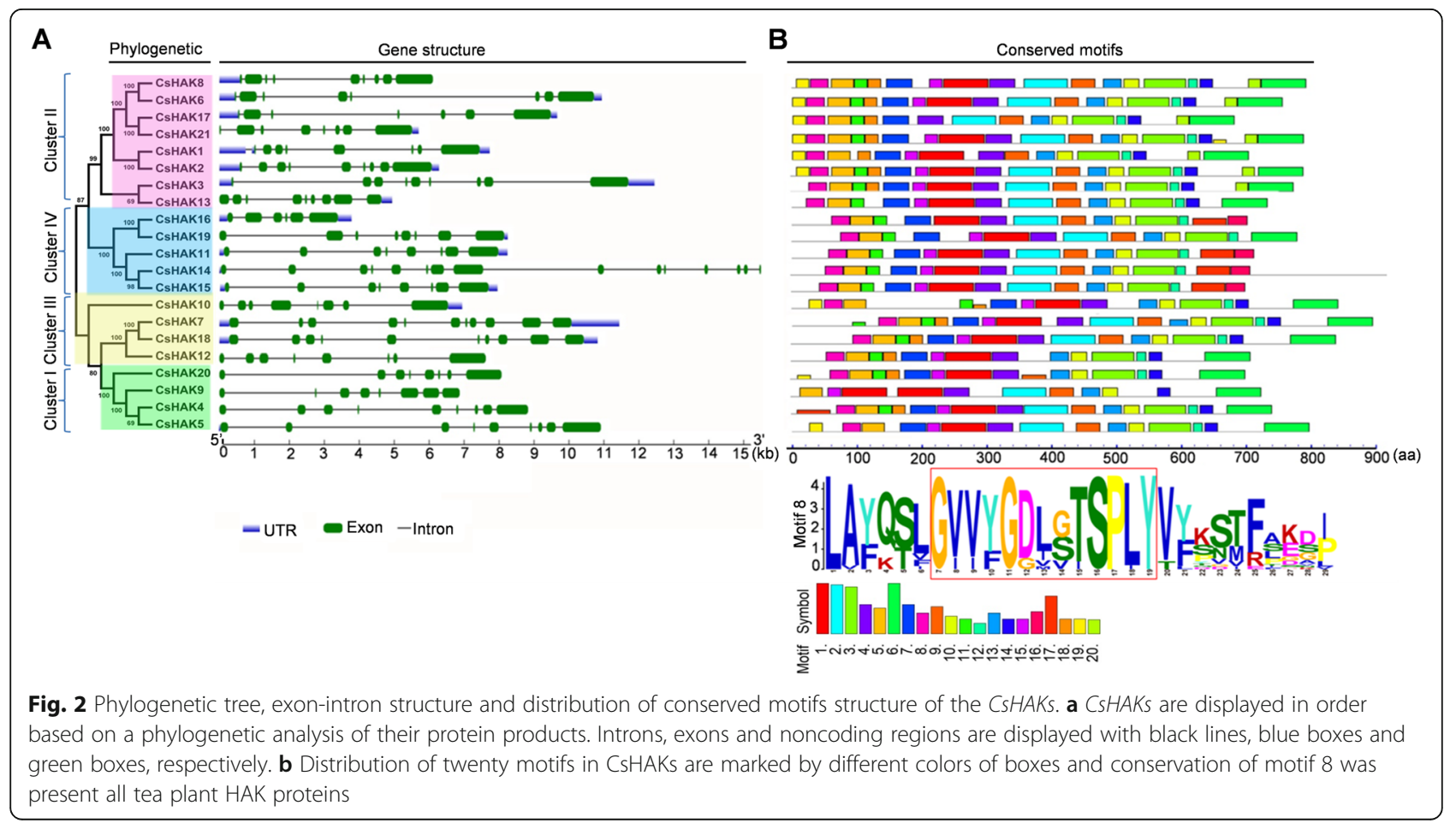




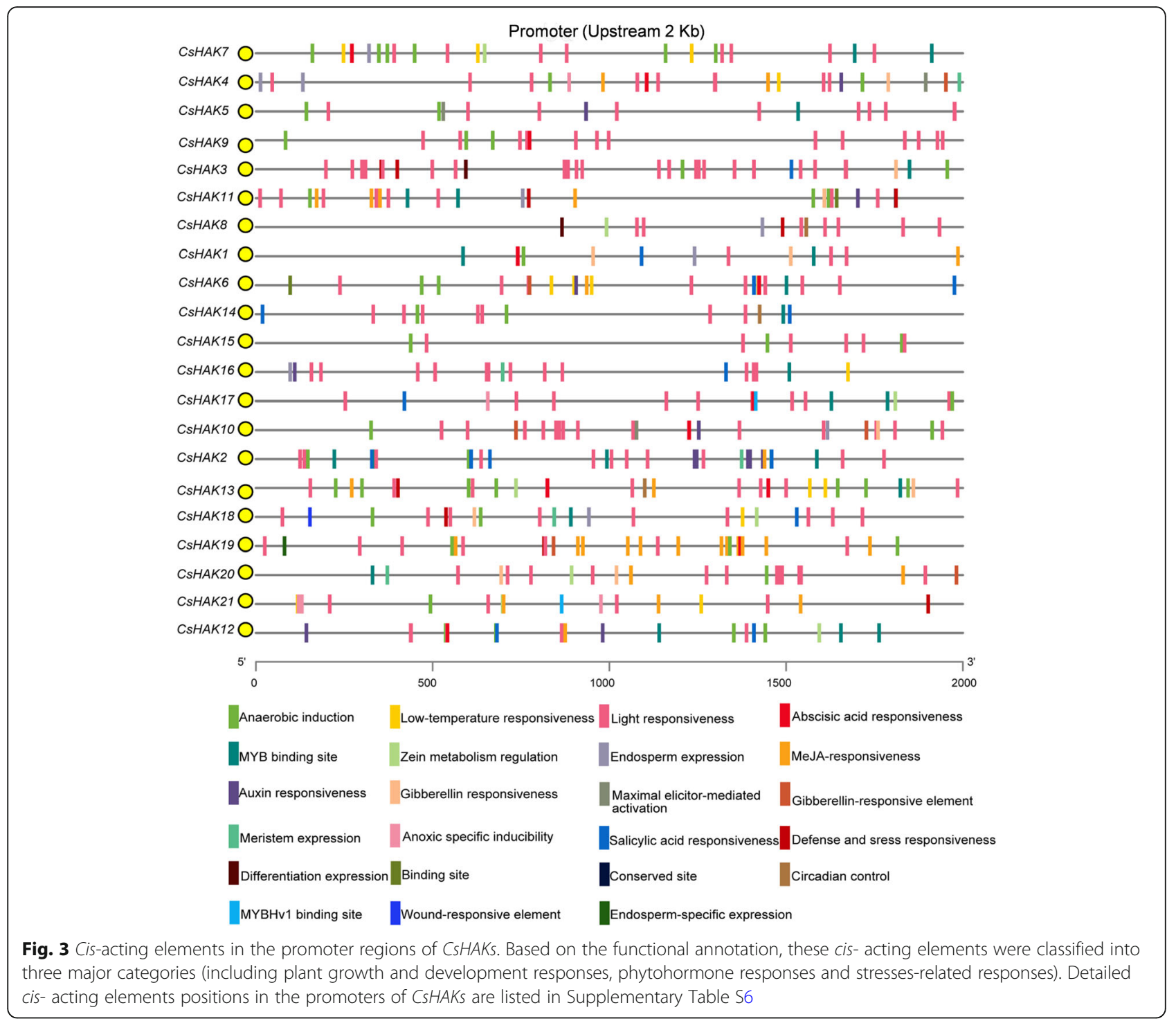

found in the promoters of CsHAKs. The CAT-box involved in meristem expression was identified in the promoters of five CsHAKs. Among the cis-acting elements belonging to hormone responses, auxin-, ethylene-, abscisic acid-, the MeJA- and gibberellin-responsive elements were observed in the promoters of eight, twenty, eleven, ten and eight CsHAKs (Fig. 3, Fig. S2, Table S5), respectively. Among stress-related responses elements, interestingly, anaerobic induction element (ARE element) was observed in the promoters of almost all CsHAKs except for CsHAK8. Moreover, stress-related (defense and wound, stress response to low-temperature and drought) cis-acting elements were also identified in the promoters of seven, one, seven and twelve CsHAKs (Fig. 3, Fig. S2, Table S5). Also, cis- acting elements responsive to one of the most important environmental factors, light were present in the promoters of all CsHAKs (Fig. 3, Fig. S2, Table S5).

\section{Tissue-specific expression of CsHAKs}

To further unveil the potential roles of CsHAKs, the tissue-specific expression of all the CsHAKs was analyzed using reliable transcriptomic database of 8 tissues of tea plant [59]. Importantly, the organs/tissues represented different developmental stages of the tea plant (Fig. 4). Hierarchical clustering was generated using the FPKM (fragments per kilobase million) values of eight tissues (detailed FPKM values are listed in Table S6) to present the relative expression levels of CsHAKs in different tissues. As shown in Fig. 4, some CsHAKs showed similar expression levels in the eight tissues, while other CsHAKs presented significant tissue-specific expression 


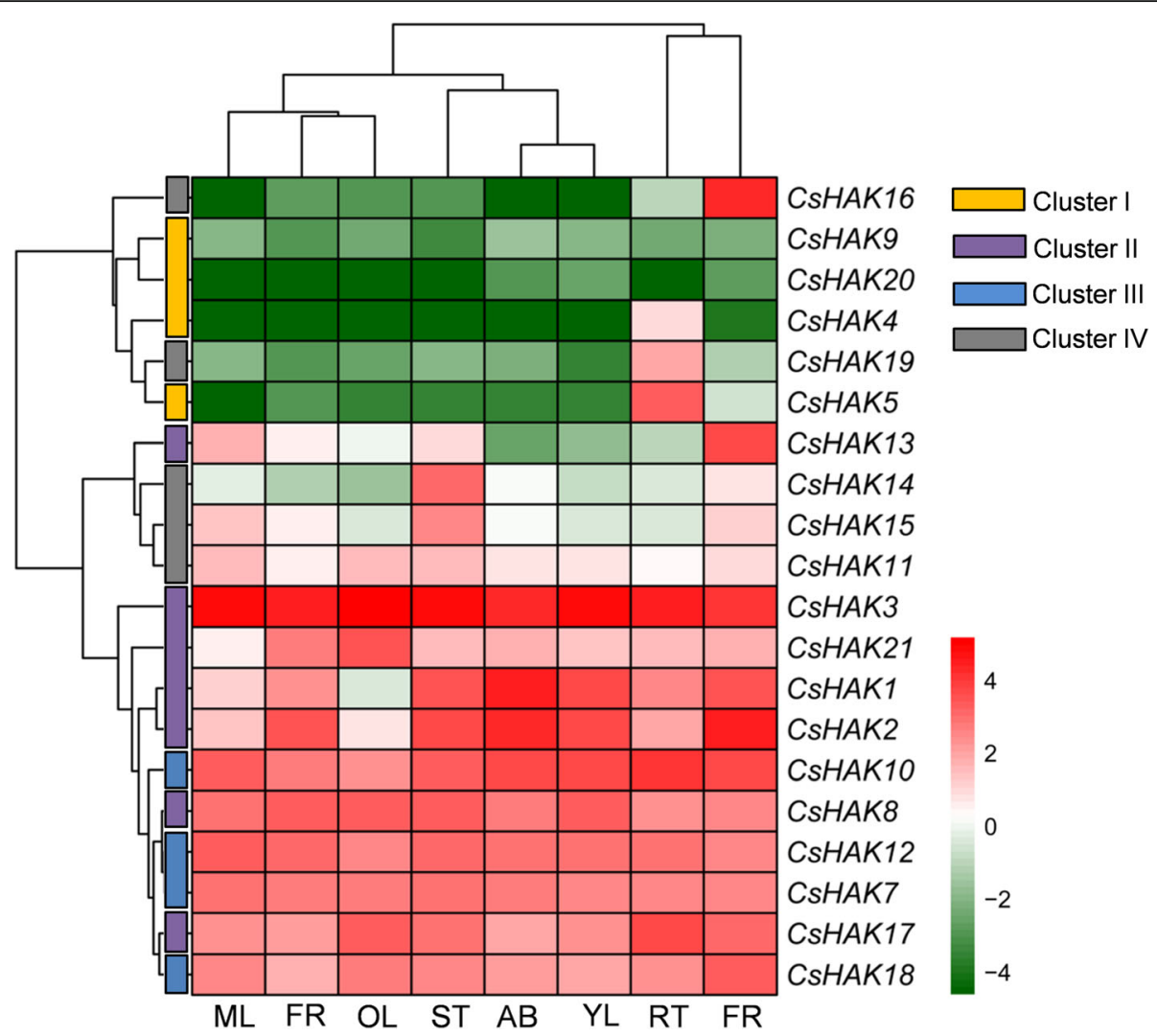

Fig. 4 Expression patterns of CSHAKs in different tea plant tissues. The color bar represents Log $_{2}$ FPKM. Red represents high expression level, green represents low expression level. The members of the CSHAKs belonging to the four clusters are represented by rectangles of different colors. The 8 tissues are apical buds (AB), young leaf $(\mathrm{YL})$, mature leaf $(\mathrm{ML})$, old leaf $(\mathrm{OL})$, young stem (ST), flower (FL), young fruit (FR) and tender root (RT). Detailed FPKM values are listed in Table S5

patterns, suggesting the functional divergence of CsHAKs among the tea plant tissues during growth and development. For example, CsHAK3 was constitutively and highly expressed in all tissues, while CsHAK9 and CsHAK20 were expressed at extremely low levels in all tissues (Fig. 4, Table S6). Notably, CsHAK4, CsHAK5, and CsHAK19 were preferentially expressed in the root, implicating them in $\mathrm{K}^{+}$acquisition from the soil (Fig. 4, Table S6). CsHAK16 was highly expressed in the flower, suggesting CsHAK16's involvement in flower development (Fig. 4, Table S6). In addition, CsHAK1 and CsHAK2 showed relatively high expression in the apical bud and young leaves, suggesting they have an important role in young shoot growth. CsHAK14 and CsHAK15 expression in the stem was relatively high, implying a possible participation in long-distance transport of $\mathrm{K}^{+}$in the tea plant. It is noteworthy that some CsHAKs (CsHAK7, $8,10,12,17,18)$ maintained a substantially high expression in all tissues (Fig. 4, Table S6), suggesting that these genes play an important role in all these tissues represented. Increasingly, studies on different plant species suggested that $H A K / K U P / K T s$ from cluster I are mainly expressed in root and play an essential role in highaffinity $\mathrm{K}^{+}$transport in plant [30, 32, 33], while HAKs from cluster II were shown to be involved in plant growth and development and were expressed in almost all plant tissues $[29,40]$. Based on these and similarity in expression patterns of CsHAKs in tea plant, we speculated that CsHAKs from the same cluster perform similar functions.

\section{The effect of $\mathrm{K}^{+}$deficiency on CsHAKs expression}

It has been extensively reported that the HAK/KUP/KT transporters are essential for $\mathrm{K}^{+}$uptake and longdistance transport in various plant species, especially under $\mathrm{K}^{+}$-limiting conditions $[20,64]$. To examine responses of CsHAKs to $\mathrm{K}^{+}$deficiency, the transcript levels of twelve selected CsHAKs from four clusters (including cluster I: CsHAK5, 20; cluster II: CsHAK1, 8, 17, 21; cluster III: CsHAK7, 10, 12, 18 and cluster IV: CsHAK11, 19) were assessed using qRT-PCR in the tea plant roots exposed to $\mathrm{K}^{+}$deficiency. On the whole, the expression 
levels of eight CsHAKs were induced by $\mathrm{K}^{+}$removal compared to control condition. However, three different expression patterns were found among these genes. The first pattern was characterized by rapid upregulation by $\mathrm{K}^{+}$removal, as seen in CsHAK7, 8, 11, 12, 18, 19, 20 (Fig. 5). The second pattern is exemplified by CsHAK21, whose expression level of which remained continually elevated during $\mathrm{K}^{+}$deficiency (as assayed at the indicated time points), except at $24 \mathrm{~h}$ (Fig. 5). The third pattern was exemplified by CsHAK5. Its expression level barely changed till $12 \mathrm{~h}$ after $\mathrm{K}^{+}$starvation, followed by a remarkable elevation at $24 \mathrm{~h}$, comparing to the control condition (Fig. 5). In contrast, the expression of CsHAK17 was constantly suppressed in the absence of
$\mathrm{K}^{+}$at all time points up to $12 \mathrm{~h}$. No significant change was observed in the expression levels of CsHAK1 and CsHAK10 after removal of $\mathrm{K}^{+}$. These results demonstrate that among the tested CsHAKs, CsHAK7, 8, 11, $12,18,20$ were highly expressed in roots, greatly responsive to $\mathrm{K}^{+}$deficiency and could be involved in $\mathrm{K}^{+}$acquisition from soil by tea plants.

\section{Effects of various stresses and phytohormones on the expression of CsHAKs}

In addition to grow in plains, most tea plants grow in the wild in mountainous and hilly areas and often face potassium starvation. Generally, $\mathrm{K}^{+}$-starved tea plants are greatly vulnerable to various stress types, both biotic

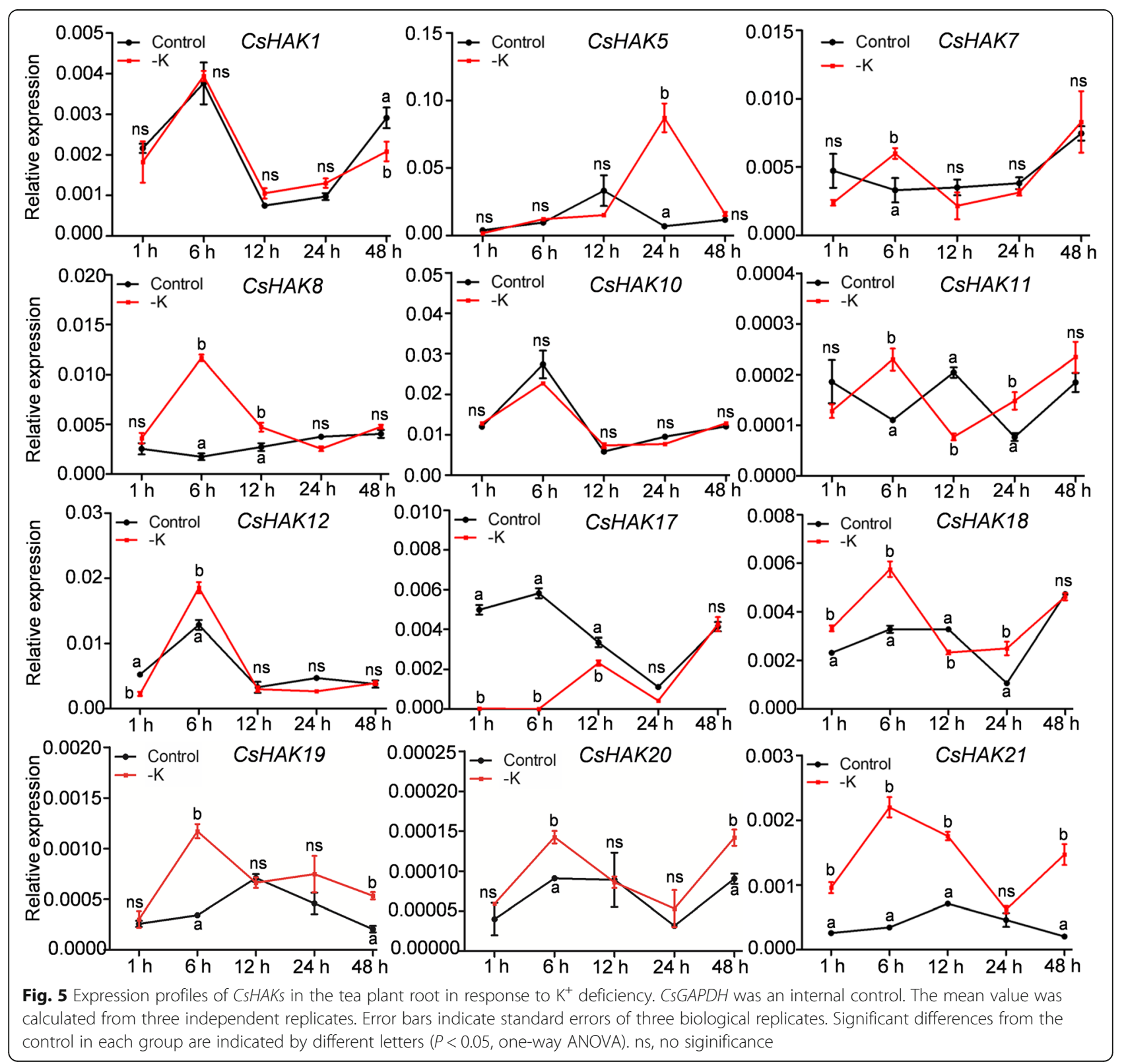


(pests, fungal diseases) and abiotic (drought, cold, high temperature) $[9,61,65]$. Potassium improves the resistance of tea plants to these stresses and promotes the synthesis and partitioning of photosynthate $[8,12$, 65]. Therefore, we further examined the response of CsHAKs expression in root to abiotic stresses and phytohormones.

$H A K / K U P / K T$ gene expression was strongly induced by salt stress, and played a crucial role in enhancing salt tolerance in other plants [30, 66]. Similarly, most of tested CsHAKs, including CsHAK1, 5, 7, 8, 10, 11, 12, were appreciably upregulated in the presence of salt stress (Fig. 6). In contrast, the expression of CsHAK17 was greatly suppressed, while that of CsHAK11,
CsHAK18 and CsHAK2O remained stable at the same conditions (Fig. 6).

Drought is a common environmental stress of the tea plant during the whole growth and development period [9]. To reveal the potential role of CsHAKs in response to dehydration, drought stress was mimicked by applying 20\% PEG6000 in the culture solutions. The expression of most of CsHAKs increased at different time points since the start of "drought" exposure, albeit in varying patterns (Fig. 7). CsHAK7 and CsHAK12 showed similar expression pattern and reached maximum at $48 \mathrm{~h}$ after PEG6000 treatment. In contrast, the expression of CsHAK5, CsHAK18 and CsHAK2O did not significantly respond to drought stress. Additionally, the expression

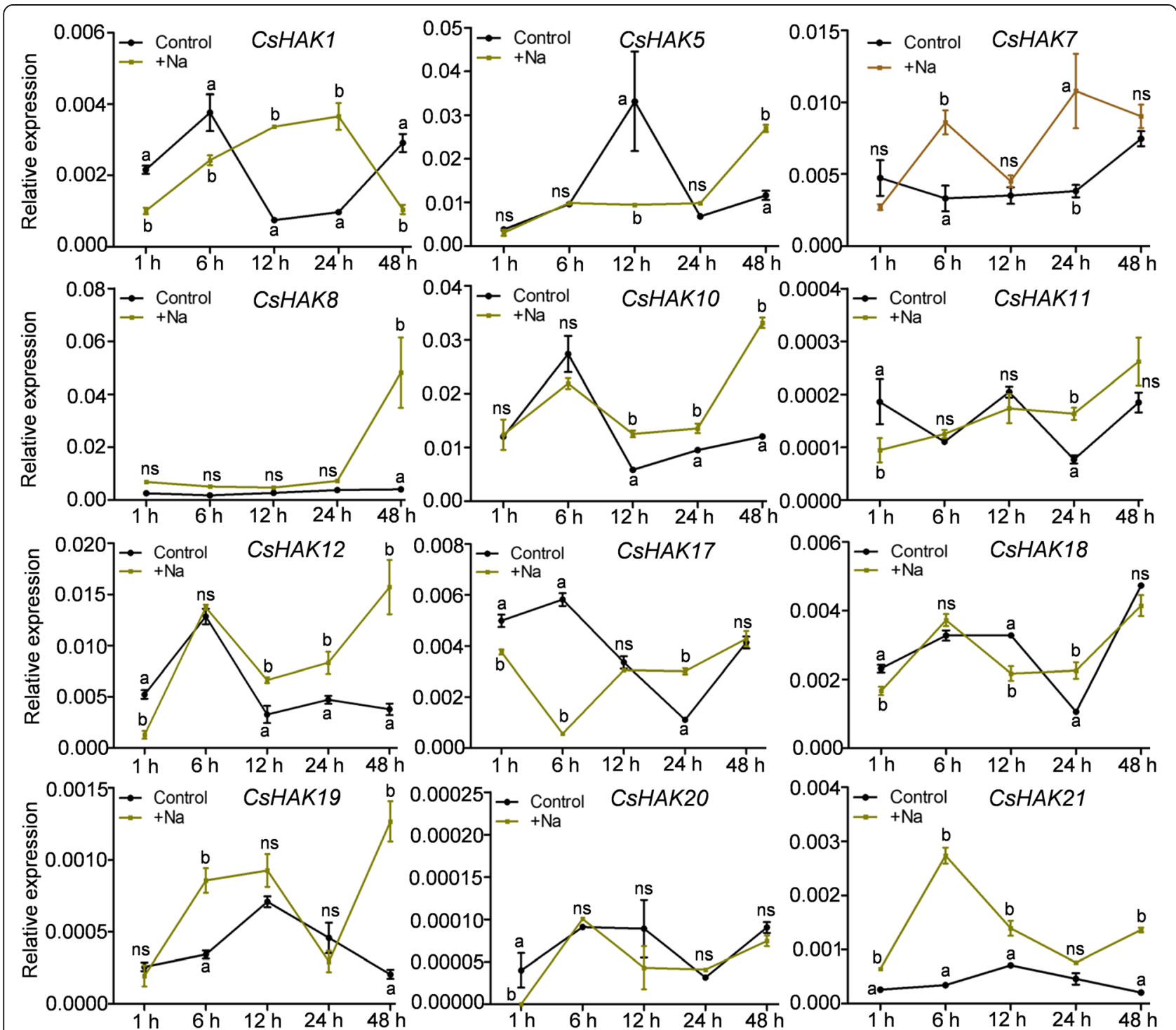

Fig. 6 Expression profiles of CSHAKs in tea plant root in response to salt stress. CsGAPDH was an internal control. The mean value was calculated from three independent replicates. Error bars indicate standard errors of three biological replicates. Significant differences from the control in each group are indicated by different letters $(P<0.05$, one-way ANOVA). ns, no siginificance 


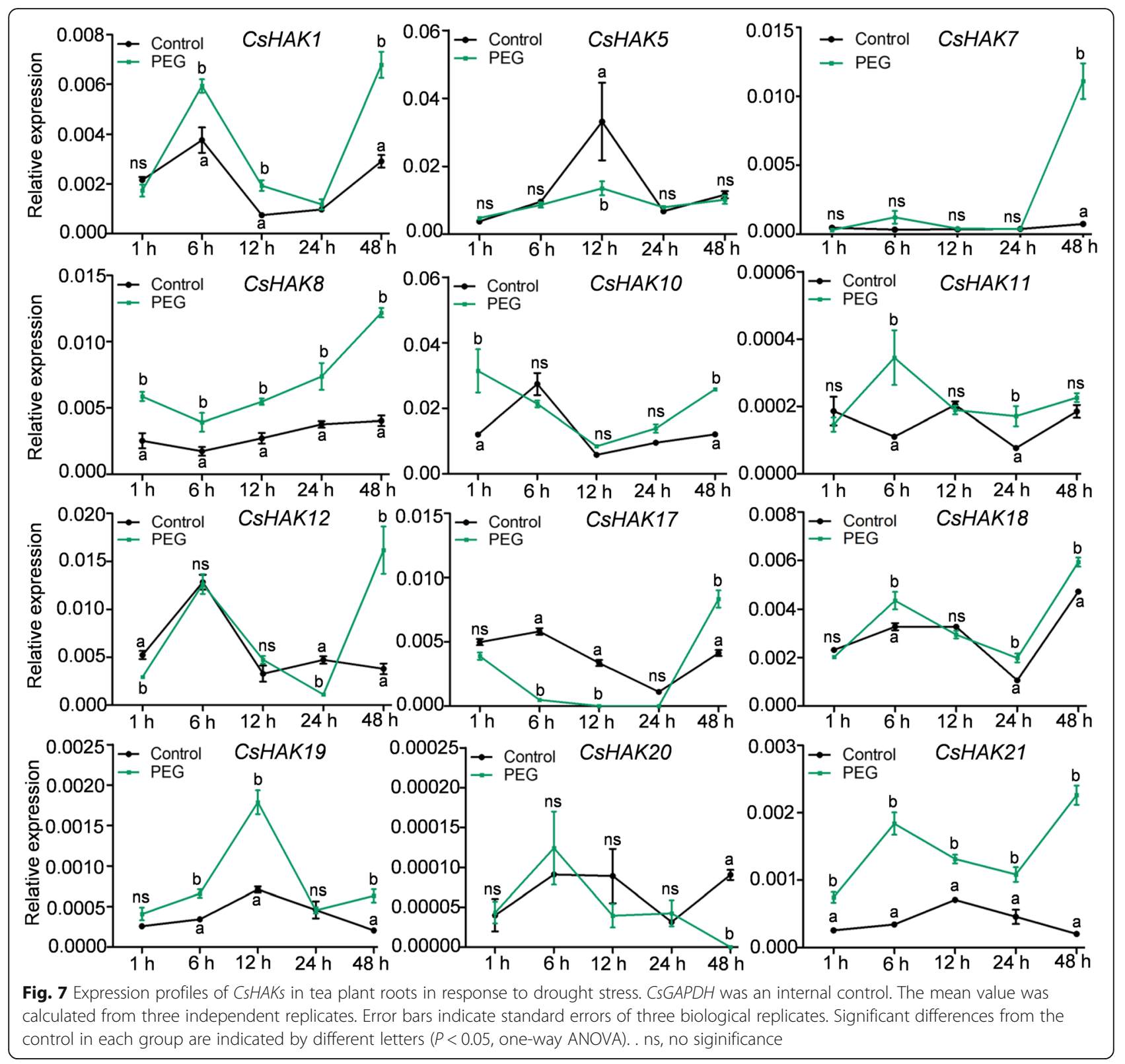

level of CsHAK17 was continuously down-regulated during the $24 \mathrm{~h}$ of exposure to the treatment, and was then moderately upregulated at $48 \mathrm{~h}$ (Fig. 7).

Previously studies showed that HAK/KUP/KT expression was regulated by phytohormones in plants $[29,38]$. Therefore, we investigated the effects of IAA and ABA on the expression of CsHAKs. Under IAA treatment, the expression of CsHAK1, 7, 8, 11, 12, 21 was markedly elevated, while the remaining CsHAKs showed no obvious induction of expression except for one or two time points (Fig. 8). The cis-acting element of auxin responsiveness was found in the promoters of CsHAK11 and CsHAK12. This element is likely to be involved in regulating CsHAK11 and CsHAK12 expression in response to
IAA treatment. ABA treatment increased the expression of five CsHAKs (CsHAK7, 8, 12, 18, 19) compared to controls and did not affect the expression of other CsHAKs (Fig. S3).

Abiotic stresses, such as salt and drought, are generally increased the accumulation of ABA [67]. The presence of multiple cis-acting elements of ABA responsiveness which we found in the promoters of the tested genes (Fig. 3) correlated with the effect of ABA on these genes expression. In addition, $C s H A K 7,8,11,12$ have IAA responsiveness elements in their promoters and were also responsive to IAA treatments. These results suggested a lot of these cis-elements in the regulation of CsHAKs by abiotic stresses, ABA and IAA. 


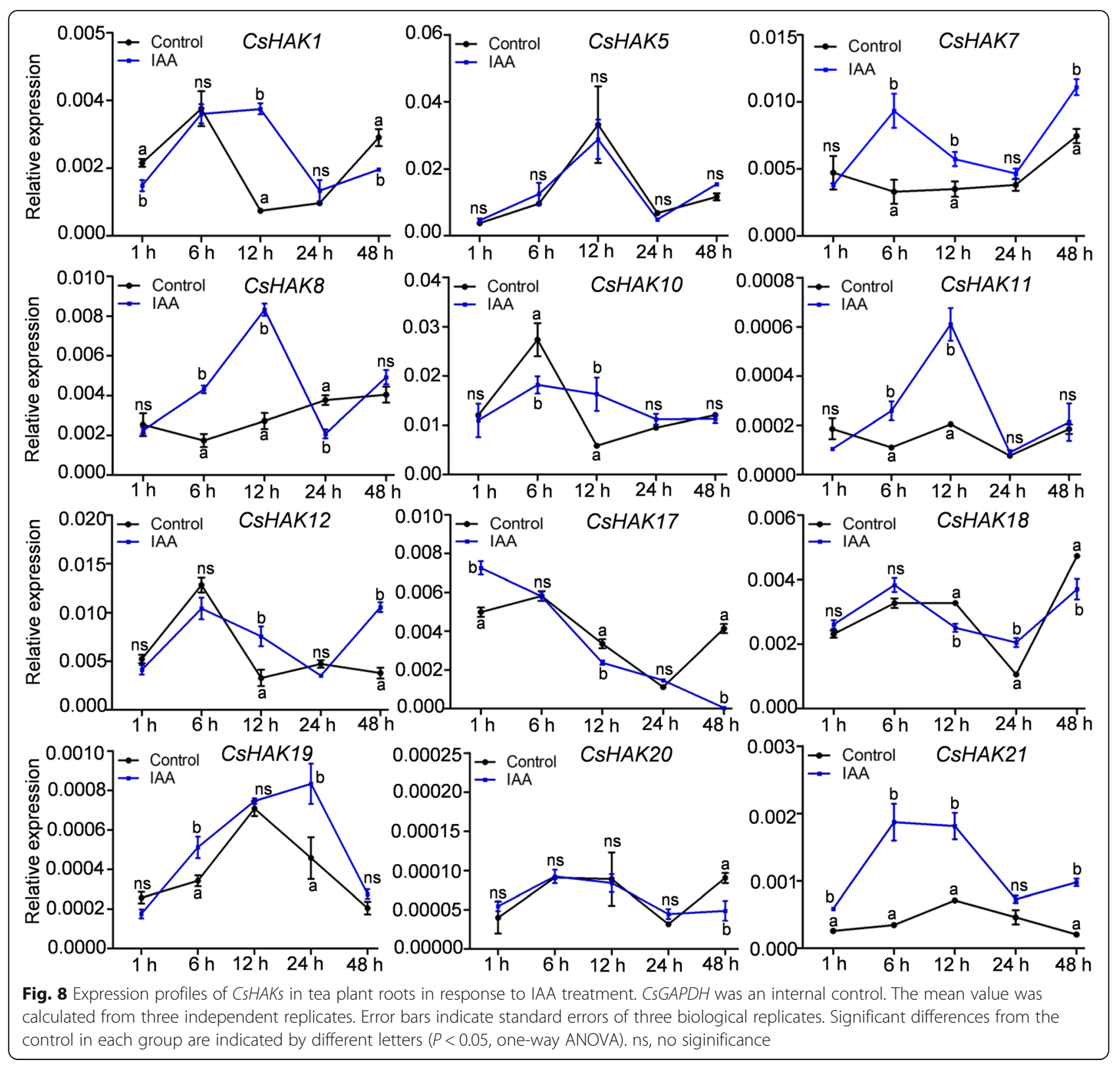

\section{Functional characterization and subcellular localization of} CsHAK7

We selected CsHAK7 for further functional characterization considering the following facts. Firstly, CsHAK7 was constitutively expressed in all eight tissues tested. Secondly, CsHAK7 expression was induced by $\mathrm{K}^{+}$ deficiency, salt and drought stresses, IAA and ABA treatments. Thirdly, within the 21 CsHAKs, CsHAK7 has the relative higher identity with the homolog in Arabidopsis (Table S2), and AtHAK7 has been well functionally characterized [52]. These facts suggested a critical role of CsHAK7 in tea plant growth and stress responses. To confirm the expression patterns of CsHAK7 in the eight tissues obtained by RNA-seq, we verified its expression patterns by qRT-PCR. As shown in Fig. 9a, CsHAK7 was constitutively and highly expressed, although not at the same level, in all the tissues tested, especially in mature leaves and buds. This was generally consistent with the transcriptome data (Fig. 4).

CsHAK7 was predicted to be located in the plasma membrane (Table 1). To verify its subcellular localization, the whole ORF without the stop codon of CsHAK7 was fused to the N-terminal of GFP reporter of pCAMBIA1305 expression vector driven by CaMV 35S promoter, generating a fusion construct CsHAK7::GFP. The CsHAK7::GFP and AtPIP2A::mCherry (plasma membrane marker gene) [68] were co-transformed into tobacco leaf epidermal cells. Microscopic visualization 


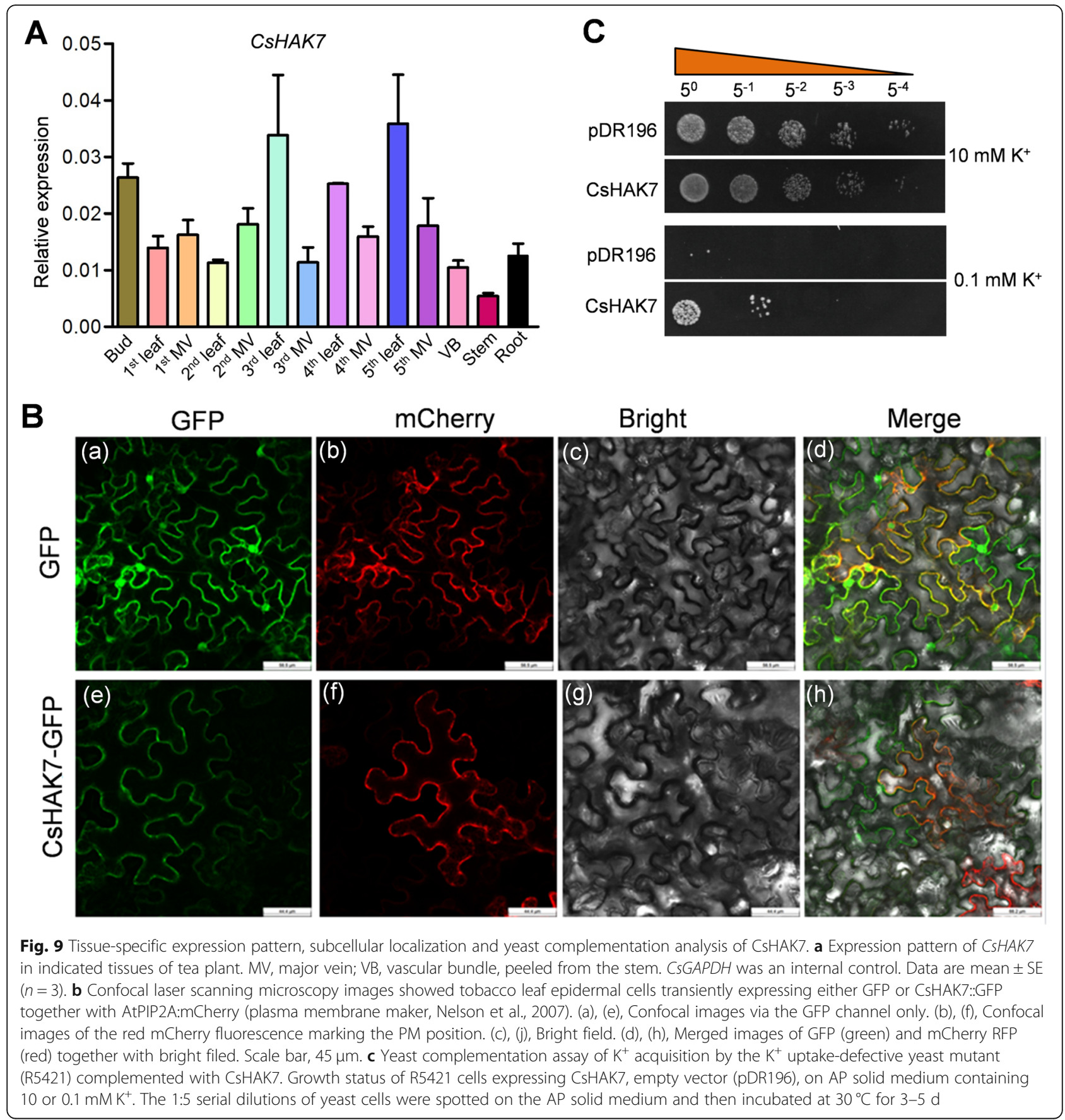

demonstrated that the fluorescence of CsHAK7-GFP overlapped with the fluorescence of AtPIP2A::mCherry (Fig. 9b). However, green fluorescence was observed in the entire cell region when the only GFP plasmid was transformed into tobacco leaf epidermal cells (Fig. 9b). The results demonstrated that CsHAK7 is localized in the plasma membrane.

To characterize the function of CsHAK7 in $\mathrm{K}^{+}$uptake, a yeast mutant complementation assay was performed. Initially, the plasmids of yeast empty vector pDR196 and
pDR196-CsHAK7 were transformed into a $\mathrm{K}^{+}$-uptakedefective strain mutant R5421 [30]. This mutant could not grow well in the presence of low concentration of $\mathrm{K}^{+}$. The yeast growth assays were conducted on solid arginine phosphate (AP) medium containing high or low $\mathrm{K}^{+}$concentrations. As shown in Fig. 9c, both yeast transformants, one with the empty vector (pDR196) and one expressing CsHAK7, could grow on high $\mathrm{K}^{+}(10 \mathrm{mM})$ medium. However, at the low $\mathrm{K}^{+}$concentration $(0.1$ $\mathrm{mM})$, the growth of the pDR196 yeast transformant was 
completely suppressed, while the growth of the CsHAK7 yeast transformant was rescued (Fig. 9c). This result suggested CsHAK7 is a functional $\mathrm{K}^{+}$transporter.

\section{Discussion}

Plant HAK/KUP/KT family is the largest gene family of $\mathrm{K}^{+}$transporters, responsible for $\mathrm{K}^{+}$acquisition and transport with the plant, especially in the case of insufficient $\mathrm{K}^{+}$ supply [20, 21]. Here, we identified 21 CsHAK genes in the tea plant $[59,60]$, analyzed their evolutionary relationships, gene structure, predicted protein motifs, cis-acting elements, expression patterns in different tissues and under various stresses. Importantly, we further functionally characterized CsHAK7 in $\mathrm{K}^{+}$-uptake yeast.

\section{Evolutionary conservation of the CsHAK family genes}

Phylogenetic analysis showed that the HAK family genes are evolutionarily conserved in the tea plant. For instance, 20 conserved motifs were identified and evenly distributed in all CsHAK proteins sequences. Notwithstanding, several motifs specific belonging to a particular cluster of CsHAKs were observed (Fig. 2b, Table S4), which could contribute to the CsHAKs divergence (Table S4). The 21 CsHAKs classification into four subgroups (Fig. 1) was consistent with that of poplar, grape rice and wheat $[38,63]$.

\section{Expression patterns of CsHAKs are generally tissue- specific in tea plants}

The location and pattern of gene expression probably reflect its function in a plant. On the whole, the expression patterns of CsHAKs are tissue-specific. Notably, several CsHAKs were expressed especially highly in roots, such as CsHAK4 (cluster I), CsHAK5 (cluster I), CsHAK19 (cluster IV) (Fig. 4), suggesting that these genes facilitate $\mathrm{K}^{+}$uptake from soil. The CsHAK16 from cluster IV was exclusively and highly expressed in flowers while in other tissues its expression levels were extremely low, suggesting that it plays a critical role in the tea plant reproductive tissue.

The number of plant $H A K / K U P / K T$ genes in clusters II and III is much larger than in clusters I and IV. This was widely observed in various plant species as well as in tea plants (Fig. 4) [38]. The expression of HAK/KUP/ $K T$ genes from clusters II and III across different tissues may explain why the $H A K / K U P / K T$ genes play multiple functions in plants [18]. Some plant $H A K / K U P / K T$ genes from clusters II and III in plants other than the tea plant not only mediated $\mathrm{K}^{+}$uptake and transport at both low and high $\mathrm{K}^{+}$supply, but also participated in plant growth and development. For example, knockout of AtKUP4 impaired root hair elongation [29] and mutation of AtKT2/KUP2 caused reduced hypocotyl length in Arabidopsis [51]. Surprisingly, these phenotypes of mutants could not be rescued in the presence of high $\mathrm{K}^{+}$ concentration. In tea plants, these genes (CsHAK1, 2, 3, $7,8,10,12,13,17,18$ and 21) from cluster II and III were also highly expressed across eight tissues (Fig. 4), implying that they also play multiple roles in the tea plant growth and development.

\section{Potential role of CsHAKs in $\mathrm{K}^{+}$deficiency and stress responses in tea plants}

Transcriptional or post-transcriptional regulation $\mathrm{K}^{+}$ transporter genes are two major mechanisms underlying responses of plants exposed to $\mathrm{K}^{+}$-limitation conditions $[19,20,69]$. Studies of various plant species revealed induction of expression of $H A K / K U P / K T$ genes by $\mathrm{K}^{+}$starvation. For instance, in Arabidopsis, AtHAK5 expression was markedly induced in roots under $\mathrm{K}^{+}$limitation $[28,46]$. In rice, the OsHAK1 and OsHAK5 were both greatly upregulated in roots after exposure to low $\mathrm{K}^{+}$ stress and they facilitated $\mathrm{K}^{+}$translocation from root to shoot at low- or high- $\mathrm{K}^{+}$concentrations [30, 32]. Recently, Qin et al. (2018) reported that the expression of $Z m H A K 1$ in maize roots increased appreciably in $\mathrm{K}^{+}$-deficiency conditions, while ZmHAK5 expression was not changed [70]. ZmHAK1 together with ZmHAK5 played a crucial role in $\mathrm{K}^{+}$uptake and translocation in maize after an exposure to $\mathrm{K}^{+}$deficiency [70]. In rice, the majority of 17 OsHAK genes were upregulated by $\mathrm{K}^{+}$starvation [71]. As expected, the expression of most tested CsHAKs was also induced after removal of $\mathrm{K}^{+}$, as assayed at the different time points, albeit to different levels (Fig. 5). It should be noted that the expression of the studied CsHAKs (CsHAK8, 12, 18, 20) was induced after a short period of $\mathrm{K}^{+}$starvation and then rapidly declined (Fig. $5)$, suggesting that these genes probably responded to the low $\mathrm{K}^{+}$signal already at an early stage. The CsHAK5, CsHAK19 and CsHAK21 showed consecutive induction (Fig. 5), implying that these genes are involved in maintaining $\mathrm{K}^{+}$homeostasis in tea plant under $\mathrm{K}^{+}$-deficient condition.

Previous studies reported that the HAK/KUP/KT genes were very responsive to various stress types and to plant hormones [35, 38, 72] and positively regulated stress responses in plants $[66,73]$. Interestingly, many cis-acting elements related to plant growth and development, stresses and phytohormone responses were extensively distributed in the promoter regions of CsHAKs (Fig. 3), implying that CsHAKs also participate in stress and phytohormone responses. In rice, OsHAK21 was significantly induced by salt stress and functioned in salt tolerance by maintaining the $\mathrm{Na}^{+} / \mathrm{K}^{+}$homeostasis [33]. The OsHAK5 transcript level also greatly increased in roots after exposure to salt stress and enhanced salt resistance of rice plants by elevating the ions ratio $\left[\mathrm{K}^{+}\right] /$ $\left[\mathrm{Na}^{+}\right]$in shoots [30]. Most of the tested CsHAKs from 
clusters I-IV were upregulated by salt stress (Fig. 6). For instance, the expression level of CsHAK7 rapidly increased at $6 \mathrm{~h}$ and reached a maximum at the time point of $24 \mathrm{~h}$, and then decreased at $48 \mathrm{~h}$ time point (Fig. 6). CsHAK1 transcript level increased continuously and stably during the first $24 \mathrm{~h}$ period and then quickly decreased at $48 \mathrm{~h}$ (Fig. 6), suggesting that CsHAK1 responded to salt stress at an early stage. These results suggest the CsHAK genes have a role in enhancing salt resistance of the tea plant.

Drought stress was a major abiotic stress commonly threatens plant survival [67]. In rice, overexpression of OsHAK1 improved tolerance to drought [34]. The drought tolerance of this transgenic rice was reportedly due to an enhancement of antioxidant enzymes and higher accumulation of a higher amount of proline [34]. We also found a few CsHAKs that were remarkably responsive to dehydration. The consistent upregulation of the transcript levels of CsHAK1, 8, 21 expression across all time points (Fig. 7), suggested their involvement in drought stress responses in tea plants.

Similar to the reported effects of phytohormones on $H A K / K U P / K T$ genes in other plants [35, 38], we found some CsHAKs were also responsive to phytohormones. This confirmed our expectations based the cis-elements of auxin and $\mathrm{ABA}$ responsiveness that we found in the promoter regions of several CsHAKs (Fig. 3). Indeed, there was a high correlation between the presence of the particular cis-elements in the gene's promoter and this gene's responsiveness to the particular phytohormone. For example: The CsHAK11 transcript level was greatly induced in roots under IAA treatment (Fig. 8) while the promoter region of CsHAK11 contained a cis-element of auxin responsiveness (Fig. 3). Also, the expression levels of CsHAK7 and CsHAK21 were rapidly upregulated under ABA treatment (Fig. S3) matching the presence of ABA-responsiveness cis-element(s) in the promoters. Interestingly, the upregulated expression of these genes in the roots of tea plant, echoes the impairment of $\mathrm{ABA}$ responses in Arabidopsis lateral root cells due to a mutation of AtKUP2/6/8 [29]. Incidentally, this Arabidopsis mutant also had enhanced cell expansion and impaired ABA responses in guard cells [29], all of which help link the effect of ABA on the CsHAKs expression to $\mathrm{ABA}$ regulation of $\mathrm{K}^{+}$homeostasis in the whole tea plant.

\section{CsHAK7 is a potentially key gene for $\mathrm{K}^{+}$acquisition in the tea plant}

Recently, the expression pattern and functional characterization of AtHAK7 was investigated [52]. The results of this study showed that AtKUP7 was expressed in all tissues, including root, leaf, stem, flower, and silique, especially a higher expression level in stelar tissues and was shown to be involved in transport $\mathrm{K}^{+}$into xylem under $\mathrm{K}^{+}$-limited conditions [52]. AtKUP7 shares almost highest amino acids identify with CsHAK7 in tea plant. Quantitative results showed CsHAK7 also expressed in all tissues of tea plant, especially a relatively higher in mature leaves and main vein (Fig. 9), suggested a similarity in their function. Indeed, the rescue by CsHAK7 of the $\mathrm{K}^{+}$-transport-disabled yeast mutant in a $\mathrm{K}^{+}$-deficient medium suggests that CsHAK7 functions in a high-affinity $\mathrm{K}^{+}$uptake, resembling the demonstrated involvement of AtKUP7 in $\mathrm{K}^{+}$transport into xylem under $\mathrm{K}^{+}$-limitation [52]. Based on the relatively higher CsHAK7 expression in mature leaves and main vein (Fig. 9), we speculate that CsHAK7 plays a critical role in $\mathrm{K}^{+}$ transfer under $\mathrm{K}^{+}$-deficiency conditions from mature leaf to new shoots in the tea plant. This function could be perhaps related to the general mechanism underlying the known phenomenon of $\mathrm{K}^{+}$mobilization from source leaves to young developing leaves temporarily overcoming $\mathrm{K}^{+}$deficiency and delaying the appearance of its symptoms in the leaves [74, 75]. Based on the upregulation of CsHAK7 expression by salt and drought stresses, ABA treatment, and by $\mathrm{K}^{+}$deficiency, we speculate that CsHAK7 fine-tunes $\mathrm{K}^{+}$fluxes across the tea plant plasma membrane to adjust the turgor pressure as a part of the plant's stress tolerance. Moreover, CsHAK7 expression was also induced by IAA, suggested a role of CsHAK7 in tea plant growth. Other functions of CsHAK7 and its physiological role in the tea plant warrant future in-depth exploration.

\section{Conclusions}

In conclusion, a total of 21 CsHAK family members were identified in tea plants. Based on the phylogenetic and structural features analysis, all 21 CsHAKs were classified into four clusters (I-IV). cis-acting elements related to plant growth and development, stresses and plant hormone were found in the CsHAKs promoter regions. The analysis of issue-specific and various stress typesinduced expression patterns suggested that CsHAKs function in $\mathrm{K}^{+}$uptake and stress responses in the tea plant root. Importantly, we demonstrated CsHAK7 participation in $\mathrm{K}^{+}$uptake in yeast. Taken together, our findings offer a good platform for further characterization of the multiple physiological roles of CsHAKs in the tea plant.

\section{Methods}

\section{Identification CsHAKs in tea plants}

To identify and annotate CsHAKs in tea plants, both the Hidden Markov Model (HMMER) profile and local BLAST searches were combined to analyze the genomic data. Firstly, the HMM profile (PF02705) [76] of the CsHAK proteins conserved domain was used to screen 
protein sequences from tea plant (Camellia sinensis vs 'Shuchazao') genome [59]. Secondly, 13 Arabidopsis AtKUP/HAK/KT protein sequences and 27 rice OsHAK protein sequences were used (as described previously for Arabidopsis [28] and for rice [38]) as queries to screen against the tea plant genome database with BLASTP program (e-value $<1 \mathrm{e}^{-5}$ ). Finally, the results of the two methods were merged to obtain candidate CsHAK family members and further verified for the presence of complete CsHAK domains by screening against the CCD (https://www.ncbi.nlm.nih.gov/cdd/), InterProScan [77] and SMART [78] databases. The physical and chemical parameters of the CsHAK proteins, i.e., their molecular weights and isoelectric points were calculated by the ExPasy website (https://web.expasy.org/protparam/). The subcellular localization of the CsHAK proteins was predicted using WoLFPSORT (http://www.genscript.com/ psort/wolf_ psort.html). The amino acid lengths of the CsHAK proteins were obtained from the genomic website $[59,60]$. Their transmembrane domains were predicted by TMHMM2 program (www. cbs.dtu.dk/services/TMHMM/).

\section{Phylogenetic tree construction of CsHAKs}

The protein sequences of HAKs in other plant species were retrieved from the NCBI database (https://www. ncbi.nlm.nih.gov/) and were used to perform multiple sequence alignments by ClustalW program (Version 2.1; http://www.clustal.org/). Phylogenetic trees were constructed based on the protein sequences of 92 HAKs using the neighbor-joining method of the program MEGA6.0 with bootstrap (1000 replicates) analysis [79]. Moreover, another phylogenetic tree was also constructed using 21 protein sequences of CsHAKs for further analysis. The genes loci of $H A K s$ in other plant species are listed in Table S2.

\section{Analysis of gene structure, motifs distribution and cis- acting elements of the CsHAKs}

The gene structure display server (GSDS) 2.0 program (http://gsds.cbi.pku.edu.cn/) was used to analyze the CsHAKs gene structure. The conserved motifs of the CsHAK proteins were predicted using MEME program (http://meme.nbcr.net/meme/cgi-bin/meme.cgi) by submitting the predicted protein sequences. The parameters of MEME were used as follows: maximum number of motifs, 20; minimum motif width, 6; and maximum motif width, 70. In addition, the promoter sequences of 2000 bp upstream of the transcription start sites of each CsHAKs were retrieved from tea plant genome website [60], and the $2000 \mathrm{bp}$ promoter regions of CsHAKs were analyzed in the PlantCARE program (http://bioinformatics.psb.ugent.be/webtools/plant care/html/).

\section{Expression profiles of CsHAKs detected by transcriptome data}

For tissue-specific expression of CsHAKs analysis, the raw transcriptome sequencing data (SRA accession no. SRP056466) [59] from eight tea plant tissues (including root, stem, apical bud, young leaf, mature leaf, old leaf, flower and fruit) were downloaded from the Sequence Read Archive (SRA) database (https://www.ncbi.nlm.nih. gov/sra/). Next, we mapped the raw transcriptome sequencing data to the tea plant genome and calculated the FPKM value according to the previous methods [59], and then the expression levels of CsHAKs were visualized by the "pheatmap" package implemented in $\mathrm{R}$ (https://cran.r-project.org/web/packages/pheatmap/ index.html).

\section{Plant material and growth conditions}

Two-year-old tea cutting seedings (Camellia sinensis L. cv. 'shuchazao') were obtained from Dechang Tea Fabrication Base at Shucheng County (Anhui province, China). Tea seedlings with uniform size were used for hydroponic culture. Tea seedlings were grown in full basal nutrient solution in a growth chamber at state Key Laboratory of Tea Plant Biology and Utilization, Anhui Agricultural University (Hefei, China) for 1 month to produce well developed roots. The growth condition of tea plants was controlled as following: light intensity of $200 \mu \mathrm{mol}$ phtotons $\mathrm{m}^{-2} \mathrm{~s}^{-1}$ for $14 \mathrm{~h}$ per day, day/light temperature of $25 / 22^{\circ} \mathrm{C}$ and relative humidity of $70 \%$. The composition of the basal nutrient solution was as reported previously [80]. The $\mathrm{pH}$ of the nutrient solution was adjusted to 4.5 , and it was replaced once a week.

\section{Plant treatments}

For $\mathrm{K}^{+}$depletion experiments, tea cutting seedlings were grown in a solution containing $0.513 \mathrm{mM} \mathrm{K}^{+}$for 1 month and then transferred into a treatment solution in which $0.2065 \mathrm{mM} \mathrm{K}_{2} \mathrm{SO}_{4}$ and/or $0.1 \mathrm{mM} \mathrm{KH}_{2} \mathrm{PO}_{4}$ was replaced by $0.2065 \mathrm{mM} \mathrm{Na}_{2} \mathrm{SO}_{4}$ and/or $0.1 \mathrm{mM} \mathrm{NaH} \mathrm{PO}_{4}$. For salinity stress experiments, the treatment solution consisted of the full basal nutrient solution supplemented with $200 \mathrm{mM} \mathrm{NaCl}$; the $\mathrm{EC}$ of the treatment solution was $17.77 \mathrm{~ms} / \mathrm{cm}$ and the control is $0.465 \mathrm{~ms} / \mathrm{cm}$; for drought stress experiment, the full basal nutrient solution contained 20\% (g/v) polyethylene glycol 6000 (PEG 6000) [68]. For experiments with phytohormones, the treatment solution was the full basal nutrient solution with added $100 \mu \mathrm{M}$ ABA or $10 \mu \mathrm{M}$ IAA [81]. After treatments, tissue samples were collected at different time points, $1 \mathrm{~h}, 6 \mathrm{~h}, 12 \mathrm{~h}, 24 \mathrm{~h}$ and $48 \mathrm{~h}$, and were immediately frozen in liquid nitrogen and stored at $-80{ }^{\circ} \mathrm{C}$ for further analysis.

For tissue-specific expression analysis, we collected different tea plant tissues: leaf, root, stem, apical bud, 
MV (major vein) and VB (vascular bundle, peeled from the stem), as described previously [58].

\section{Gene expression analysis using qRT-PCR}

Total RNAs from the various sampled tissues were isolated using the modified CTAB method reported previously [80]. First-strand cDNAs were synthesized using the PrimeScript RT Reagent Kit with gDNA Eraser (Takara, Dalian, China) according to the manufacturer's protocol. qRT-PCR was run on a Bio-Rad CFX96 machine with SYBR ${ }^{\circ}$ Premixm Ex Taq ${ }^{\text {Tm }}$ (TaKaRa, Japan). CsGAPDH (TEA025584.1) was used as an internal control. Data were analyzed with Opticon monitor software (Bio-Rad). All primers for qRT-PCR were designed using Primer 5.0 software and primer sequences are listed in Table S1. All the experiments were performed with three biological replicates. The $2^{-\Delta C T}$ method was used to calculate the relative expression level $[82,83]$.

\section{Subcellular localization analysis of CsHAK7}

The full-length coding sequence of CsHAK7 without the stop codon was amplified by RT-PCR using primers (Table S1) containing double restriction sites of SpeI and BamHI. The purified PCR products were digested with SpeI and BamHI, and then fused to the pCAMBIA1305.1:GFP expression vector to generate a fusion protein CsHAK7::GFP, driven by the CaMV 35S promoter. After validation by sequencing, the plasmids of both CsHAK7::GFP and AtPIP2A::mCherry (Plasma membrane (PM) marker) [68] or both 35S:GFP (as a control) and 35S:AtPIP2A::mCherry, were cotransformed into the Agrobacterium tumefaciens strain EHA105. The bacterial cells were collected by centrifugation and resuspended in a solution ( $\mathrm{pH}$ 5.7) containing $10 \mathrm{mM}$ MES, $10 \mathrm{mM} \mathrm{MgCl}_{2}$, and $200 \mathrm{mM}$ acetosyringone (AS). Next, a Nicotiana benthamiana plant with four to five leaves (approximately 1 month old) was used for Agrobacterium transient transformation experiment. Cell suspensions at an optical density of 0.3 (at $600 \mathrm{~nm}$ ) were infiltrated into the leaves of Nicotiana benthamiana using a needle-free syringe. 48 or $72 \mathrm{~h}$ after infiltration, we examined the tobacco epidermis cells by confocal laser scanning microscope (LSM410; Carl Zeiss); the green GFP fluorescence was elicited by excitation at $488 \mathrm{~nm}$ and observed via a $535 \mathrm{~nm}$ filter, and the red fluorescence of mCherry was elicited by excitation at $543 \mathrm{~nm}$ and observed via a $585 \mathrm{~nm}$ filter, as previously described [84].

\section{Yeast complementation assay}

To elucidate the function of CsHAK7 in $\mathrm{K}^{+}$uptake in yeast, the coding sequence of CsHAK7 was amplified with RT-PCR using primers (Table S1), and then the purified PCR products were inserted into the yeast expression vector pDR196 under the control of the inducible PMA promoter [85]. The plasmids of the expression vectors (pDR196 and pDR196-CsHAK7) were transformed into the R5421 strain, a $\mathrm{K}^{+}$-uptake-defective strain of Saccharomyces cerevisiae [30]. The yeast cell transformation method was employed as described previously [84]. Arginine phosphate (AP) medium was used for subsequent growth assays that were carried out as described previously [30, 62]. The yeast complementation tests were performed on solid AP medium, and the plates were incubated at $30{ }^{\circ} \mathrm{C}$ in dark condition for 3-5 d.

\section{Supplementary information}

Supplementary information accompanies this paper at https://doi.org/10. 1186/s12864-020-06948-6.

Additional file 1: Fig. S1. The predicted transmembrane helices of the CsHAKs. The transmembrane domains of CsHAKs proteins were predicted using an internet server, TMHMM2(www.cbs.dtu.dk/services/TMHMM/), and the red peaks represent the predicted transmembrane regions of proteins.

Additional file 2: Fig. S2. Cis-elements in promoters of CsHAKs in tea plants.

Additional file 3: Fig. S3. Expression profiles of CSHAKs in the tea plant root in response to ABA treatment. The mean value was calculated from three independent replicates. Error bars indicate standard errors of three biological replicates. Significant differences from the control in each group are indicated by different letters $(P<0.05$, one-way ANOVA). ns, no siginificance.

Additional file 4: Table S1. List of primers used in this study.

Additional file 5: Table S2. HAK/KUP/KT family genes in Arabidopsis thaliana, Oryza sativa, Populus trichocarpa, Vitis vinifera

Additional file 6: Table S3. Information of the conserved motifs in CsHAKs.

Additional file 7: Table S4. Conserved motifs identified from the CSHAKs in tea plants.

Additional file 8: Table S5. Cis-acting elements identified from the CsHAKs promoters of tea plants.

Additional file 9: Table S6. Expression levels of CSHAKs in different tissues of tea plants.

\section{Abbreviations}

HAK/KUP/KT: (High-affinity $\mathrm{K}^{+}$transporter / K uptake permease / $\mathrm{K}^{+}$ transporter); TM: Transmembrane; GFP: Green fluorescent protein; RFP: Red fluorescent protein; MW: Molecular weight; pl: Isoelectric points; TAIR: The arabidopsis information resource; GSDS: Gene structure display server; RNAseq: RNA sequencing; FPKM: Fragments per kilobase million; PM: Plasma membrane; ABA: Abscisic acid; IAA: Indole-3-acetic acid; NAA: 1naphthylacetic acid; PEG 6000: Polyethylene glycol 6000; SRA: Sequence read archive; MV: Major vein; VB: Vascular bundle; AP: Arginine phosphate.

\section{Acknowledgments}

We are grateful to Professor Nava Moran from Hebrew University of Jerusalem for her constructive comments and language editing to the manuscript, and to Dr. Rick Gaber from Northwestern University for providing the yeast mutant R5421, and to Dr. Shengrui Liu from Anhui Agricultural University for his constructive comments on this work. We would like to thank the Tea Plant Cultivar and Germplasm Resource Garden in Guohe Town, Anhui Agricultural University for providing materials of tea plants. 


\section{Authors' contributions}

T.Y., Z.Z., and X. W conceived and designed the research. Y.T., X.L., Y.W., Y.X., J.M., X.C., and E.X. performed the experiments. T.Y. analyzed the data and wrote the manuscript. Z.Z. and X.W. revised the manuscript. All authors have read and approved the final version of this manuscript.

\section{Funding}

This project was financially supported by National Natural Science Foundation of China (Grant no. 31800584) to T.Y., Anhui Provincial Department of Science and Technology (17030701049) to Z.Z., Anhui Provincial Postdoctoral Science Foundation (Grant no. 2017B158) to T.Y., Anhui Provincial Natural Science Foundation (Grant no. 1808085QC75) to T.Y. The funding bodies played no role in the design of the study or collection, analysis and interpretation of data and in writing this manuscript.

\section{Availability of data and materials}

All data generated or analyzed during this study were included in this published article and the additional files.

\section{Ethics approval and consent to participate}

Not applicable.

\section{Consent for publication}

Not applicable.

\section{Competing interests}

The authors declare that they have no competing interests.

Received: 12 March 2020 Accepted: 24 July 2020

Published online: 13 August 2020

\section{References}

1. Yu Z, Yang Z. Understanding different regulatory mechanisms of proteinaceous and non-proteinaceous amino acid formation in tea (Camellia sinensis) provides new insights into the safe and effective alteration of tea flavor and function. Crit Rev Food Sci. 2020;60(5):844-58.

2. Saeed M, Naveed M, Arif M, Kakar MU, Manzoor R, Abd El-Hack ME, Alagawany M, Tiwari R, Khandia R, Munjal A, et al. Green tea (Camellia sinensis) and L-theanine: medicinal values and beneficial applications in humans-a comprehensive review. Biomed Pharmacother. 2017;95:1260-75.

3. $\mathrm{Xu} \mathrm{J,} \mathrm{Xu} \mathrm{Z,} \mathrm{Zheng} \mathrm{W.} \mathrm{A} \mathrm{review} \mathrm{of} \mathrm{the} \mathrm{antiviral} \mathrm{role} \mathrm{of} \mathrm{green} \mathrm{tea} \mathrm{catechins.}$ Molecules. 2017;22:1337.

4. Su H, Zhang X, He Y, Li L, Wang Y, Hong G, Xu P. Transcriptomic analysis reveals the molecular adaptation of three major secondary metabolic pathways to multiple macronutrient starvation in tea (Camellia sinensis). Genes. 2020;11:241.

5. Yang T, Li H, Tai Y, Dong C, Cheng X, Xia E, et al. Transcriptional regulation of amino acid metabolism in response to nitrogen deficiency and nitrogen forms in tea plant root (Camellia sinensis L.). Sci Rep. 2020;10:6868.

6. Liu S, An Y, Tong W, Qin X, Samarina L, Guo R, Xia X, Wei C. Characterization of genome-wide genetic variations between two varieties of tea plant (Camellia sinensis) and development of InDel markers for genetic research. BMC Genomics. 2019;20(1):935.

7. Liu L, Lin N, Liu X, Yang S, Wang W, Wan X. From chloroplast biogenesis to chlorophyll accumulation: the tnterplay of light and hormones on gene expression in Camellia sinensis cv shuchazao leaves. Front Plant Sci. 2020;11:256.

8. Ruan J, Wu X, Härdter R. Effects of potassium and magnesium nutrition on the quality components of different types of tea. J Sci Food Agric. 1999;79:47-52.

9. Zhang X, Wu H, Chen J, Chen L, Wan X. (2020): chloride and amino acids are associated with $\mathrm{K}^{+}$-alleviated drought stress in tea (Camellia sinesis L.). Funct. Plant Biol. 2020;47:398-408.

10. Leigh RA, Jones W. A hypothesis relating critical potassium concentrations for growth to the distribution and functions of this ion in the plant cell. New Phytol. 1984;97:1-13.

11. Wang $M$, Zheng $Q$, Shen $Q$, Guo $S$. The critical role of potassium in plant stress response. Int J Mol Sci. 2013;14(4):7370-90.

12. Ruan J, Ma L, Shi Y. Potassium management in tea plantations: its uptake by field plants, status in soils, and efficacy on yields and quality of teas in China. J Plant Nutr Soil Sc. 2013;176(3):450-9.
13. Ruan J, Wu X. Productivity and quality response of tea to balanced nutrient management including K and Mg. J Tea Sci. 2003;23:21-6. (In Chinese).

14. Maathuis FJM. Physiological functions of mineral macronutrients. Curr Opin Plant Biol. 2009:12(3):250-8.

15. Walker DJLR, Miller AJ. Potassium homeostasis in vacuolate plant cells. Proc Natl Acad Sci U S A. 1996:93:10510-4.

16. Epstein E, Rains DW, Elzam OE. Resolution of dual mechanisms of potassium absorption by barley roots. Proc Natl Acad Sci U S A. 1963;5:684-92.

17. Very AA, Sentenac $\mathrm{H}$. Molecular mechanisms and regulation of $\mathrm{K}^{+}$transport in higher plants. Annu Rev Plant Biol. 2003;54:575-603.

18. Grabov A. Plant KT/KUP/HAK potassium transporters: single family - multiple functions. Ann Bot. 2007:99(6):1035-41.

19. Wang Y, Wu W. Potassium transport and signaling in higher plants. Annu Rev Plant Biol. 2013;64:451-76.

20. Li W, Xu G, Alli A, Yu L. Plant HAK/KUP/KT K $K^{+}$transporters: function and regulation. Semin Cell Dev Biol. 2018;74:133-41.

21. Vérya AA, Nieves-Cordonesa M, Daly M, Khana I, Fizamesa C, Sentenaca H. Molecular biology of $\mathrm{K}^{+}$transport across the plant cell membrane what do we learn from comparison between plant species. J Plant Physiol. 2014;171:748-69.

22. Nieves-Cordones M, Ródenas R, Chavanieu A, Rivero RM, Martinez V, Gaillard I, Rubio F. Uneven HAK/KUP/KT protein diversity among angiosperms: species distribution and perspectives. Front Plant Sci. 2016;7:127.

23. Zhang Z, Zhang J, Chen Y, Li R, Wang H, Wei J. Genome-wide analysis and identification of HAK potassium transporter gene family in maize (Zea mays L.). Mol Biol Rep. 2012;39(8):8465-73.

24. He C, Cui K, Duan A, Zeng Y, Zhang J. Genome-wide and molecular evolution analysis of the poplar KT/HAK/KUP potassium transporter gene family. Ecol Evol. 2012:2(8):1996-2004.

25. Hyun TK, Rim Y, Kim E, Kim JS. Genome-wide and molecular evolution analyses of the KT/HAK/KUP family in tomato (Solanum lycopersicum L.) Genes Genomics. 2014;36(3):365-74.

26. Rehman HM, Nawaz MA, Shah ZH, Daur I, Khatoon S, Yang SH, Chung G. Indepth genomic and transcriptomic analysis of five $\mathrm{K}^{+}$transporter gene families in soybean confirm their differential expression for nodulation. Front Plant Sci. 2017;8:804.

27. Li Y, Peng L, Xie C, Shi X, Dong C, Shen Q, Xu Y. Genome-wide identifcation, characterization, and expression analyses of the HAK/KUP/KT potassium transporter gene family reveals their involvement in $\mathrm{K}^{+}$deficient and abiotic stress responses in pear rootstock seedlings. Plant Growth Regul. 2018:85:187-98

28. Ahn SJ, Shin R, Schachtman DP. Expression of KT/KUP genes in Arabidopsis and the role of root hairs in $\mathrm{K}^{+}$uptake. Plant Physiol. 2004;134(3):1135-45.

29. Osakabe Y, Arinaga N, Umezawa T, Katsura S, Nagamachi K, Tanaka H, Ohiraki H, Yamada K, Seo SU, Abo M, et al. Osmotic stress responses and plant growth controlled by potassium transporters in Arabidopsis. Plant Cell. 2013;25(2):609-24

30. Yang T, Zhang S, Hu Y, Wu F, Hu Q, Chen G, Cai J, Wu T, Moran N, Yu L, et al. The role of a potassium transporter OsHAK5 in potassium acquisition and transport from roots to shoots in rice at low potassium supply levels. Plant Physiol. 2014;166:945-59.

31. Davies C, Shin R, Liu W, Thomas MR, Schachtman DP. Transporters expressed during grape berry (Vitis vinifera L.) development are associated with an increase in berry size and berry potassium accumulation. J Exp Bot. 2006;57(12):3209-16.

32. Chen G, Hu Q, Luo L, Yang T, Zhang S, Hu Y, Yu L, Xu G. Rice potassium transporter OsHAK1 is essential for maintaining potassium-mediated growth and functions in salt tolerance over low and high potassium concentration ranges. Plant Cell Environ. 2015;38:2747-65.

33. Shen $Y$, Shen $L$, Shen $Z$, Jing $W$, Ge H, Zhao J, Zhang W. The potassium transporter OsHAK21 functions in the maintenance of ion homeostasis and tolerance to salt stress in rice. Plant Cell Environ. 2015;38(12):2766-79.

34. Chen G, Liu C, Gao Z, Zhang Y, Jiang H, Zhu L, Ren D, Yu L, Xu G, Qian Q. OsHAK1, a high-affinity potassium transporter, positively regulates responses to drought stress in rice. Front Plant Sci. 2017;8:1885.

35. Jung JY, Shin R, Schachtman DP. Ethylene mediates response and tolerance to potassium deprivation in Arabidopsis. Plant Cell. 2009;21(2):607-21.

36. Kim EJ, Kwak JM, Uozumi N, Schroeder JI. AtKUP1: an Arabidopsis gene encoding high-affinity potassium transport activity. Plant Cell. 1998;10:51-62.

37. Nam YJ, Tran LS, Kojima M, Sakakibara H, Nishiyama R, Shin R. Regulatory roles of cytokinins and cytokinin signaling in response to potassium deficiency in Arabidopsis. PLoS One. 2012;7(10):e47797. 
38. Gupta M, Qiu X, Wang L, Xie W, Zhang C, Xiong L, Lian X, Zhang Q. KT/HAK/KUP potassium transporters gene family and their whole-life cycle expression profile in rice (Oryza sativa). Mol Gen Genomics. 2008; 280:437-52.

39. Bañuelos MA, Klein RD, Alexander-Bowman SJ, Rodríguez-Navarro A. A potassium transporter of the yeast Schwanniomyces occidentalis homologous to the KUP system of Escherichiacoli has a high concentrative capacity. EMBO J. 1995;14(13):3021-7.

40. Rigas S, Ditengou FA, Ljung K, Daras G, Tietz O, Palme K, Hatzopoulos P. Root gravitropism and root hair development constitute coupled developmental responses regulated by auxin homeostasis in the Arabidopsis root apex. New Phytol. 2013;197:1130-41.

41. Fu H, Luan S. AtKUP1: a dual-affinity $\mathrm{K}^{+}$transporter from Arabidopsis. Plant Cell. 1998;10:63-73.

42. Rubio F, Santa-María GE, Rodríguez-Navarro A. Cloning of Arabidopsis and barley cDNAs encoding HAK potassium transporters in root and shoot cells. Physiol Plant. 2000;109:34-43.

43. Rodríguez-Navarro A. Potassium transport in fungi and plants. Biochim Biophys Acta. 2000;1469:1-30

44. Gomez-Porras JL, Riano-Pachon DM, Benito B, Haro R, Sklodowski K, Rodríguez-Navarro A, Dreyer I. Phylogenetic analysis of $\mathrm{K}^{+}$transporters in bryophytes, lycophytes, and flowering plants indicates a specialization of vascular plants. Front Plant Sci. 2012;3:167.

45. Nieves-Cordones M, Alemán F, Martínez V, Rubio F. The Arabidopsis thaliana HAK5 $\mathrm{K}^{+}$transporter is required for plant growth and $\mathrm{K}^{+}$acquisition from low $\mathrm{K}^{+}$solutions under saline conditions. Mol Plant. 2010;3(2):326-33.

46. Gierth M, Mäser P, Schroeder Jl. The potassium transporter AtHAK5 functions in $\mathrm{K}^{+}$deprivation-induced high-affinity $\mathrm{K}^{+}$uptake and AKT1 $\mathrm{K}^{+}$ channel contribution to $\mathrm{K}^{+}$uptake kinetics in Arabidopsis roots. Plant Physiol. 2005;137(3):1105-14.

47. Qi Z, Hampton CR, Shin R, Barkla BJ, White PJ, Schachtman DP. The high affinity $\mathrm{K}^{+}$transporter AtHAK5 plays a physiological role in planta at very low $\mathrm{K}^{+}$concentrations and provides a caesium uptake pathway in Arabidopsis. J Exp Bot. 2008;59(3):595-607.

48. Yang $T$, Feng $H$, Zhang $S$, Xiao H, Hu Q, Chen $G$, et al. Potassium transporter OsHAK5 alters rice architecture via ATP-dependent transmembrane auxin fluxes. Plant Comm. 2020;1:10052.

49. Rigas S, Debrosses G, Haralampidis K, Francisco V-A, Feldmann KA, Grabov A, Dolan L, Hatzopoulos P. TRH1 encodes a potassium transporter required for tip growth in Arabidopsis root hairs. Plant Cell. 2001;13:139-51.

50. Vicente-Agullo F, Rigas S, Desbrosses G, Dolan L, Hatzopoulos P, Grabov A. Potassium carrier TRH1 is required for auxin transport in Arabidopsis roots. Plant J. 2004;40(4):523-35.

51. Elumalai RP, Nagpal P, Reed JW. A mutation in the Arabidopsis KT2/KUP2 potassium transporter gene affects shoot cell expansion. Plant Cell. 2002;14(1):119-31.

52. Han $\mathrm{M}$, Wu W, Wu W, Wang Y. Potassium transporter KUP7 is involved in $\mathrm{K}^{+}$ acquisition and translocation in Arabidopsis root under $\mathrm{K}^{+}$-limited conditions. Mol Plant. 2016;9(3):437-46.

53. Mondal TK, Bhattacharya A, Laxmikumaran M, Ahuja PS. Recent advances of tea (Camellia sinensis) biotechnology. Plant Cell Tissue Organ Cult. 2006;76:195-254.

54. Mukhopadhyay M, Mondal TK, Chand PK. Biotechnological advances in tea (Camellia sinensis [L.]O. Kuntze): a review. Plant Cell Rep. 2016;35:255-87.

55. Xia E, Tong W, Wu Q, Wei S, Zhao J, Zhang Z, Wei CL, Wan X. Tea plant genomics: achievements, challenges and perspectives. Hortic Res. 2020;7:7

56. Alagarsamy KSL, Wei S. Protocol: high-efciency in-planta Agrobacteriummediated transgenic hairy root induction of Camellia sinensis var. sinensis. Plant Methods. 2018;14:17.

57. Dai X, Liu Y, Zhuang J, Yao S, Liu L, Jiang X, Zhou K, Wang Y, Xie D, Bennetzen $J$, et al. Discovery and characterization of tannase genes in plants_ rolesin hydrolysis of tannins. New Phytol. 2020;226:1104-16.

58. Dong C, Li F, Yang T, Feng L, Zhang S, Li F, Li W, Xu G, Bao S, Wan X, et al. Theanine transporters identified in tea plants (Camellia sinensis L.). Plant J. 2020;101(1):57-70.

59. Wei C, Yang H, Wang S, Zhao J, Liu C, Gao L, Xia E, Lu Y, Tai Y, She G, et al. Draft genome sequence of Camellia sinensis var. sinensis provides insights into the evolution of the tea genome and tea quality. Proc Natl Acad Sci U S A. 2018;115(18):E4151-8.

60. Xia E, Li F, Tong W, Li P, Wu Q, Zhao H, Ge R, Li R, Li Y, Zhang Z, et al. Tea plant information archive: a comprehensive genomics and bioinformatics platform for tea plant. Plant Biotechnol J. 2019;17(10):1938-53.
61. Li Y, Mi X, Zhao S, Zhu J, Guo R, Xia X, Liu L, Liu S, Wei C. Comprehensive profiling of alternative splicing landscape during cold acclimation in tea plant. BMC Genomics. 2020;21(1):65.

62. Horie T, Sugawara M, Okada T, Taira K, Kaothien-Nakayama P, Katsuhara M, Shinmyo A, Nakayama H. Rice sodium-insensitive potassium transporter, OsHAK5, confers increased salt tolerance in tobacco BY2 cells. J Biosci Bioeng. 2011;111(3):346-56.

63. Cheng X, Liu X, Mao W, Zhang X, Chen S, Zhan K, Bi H, Xu H. Genome-wide identification and analysis of HAK/KUP/KT potassium transporters gene family in wheat (Triticum aestivum L.). Int J Mol Sci. 2018;19(12):3969.

64. Santa-María GE, Rubio F, Dubcovsky J, Rodríguez-Navarro A. The HAK1 gene of barley is a member of a large gene family and encodes a high-affinity potassium transporter. Plant Cell. 1997:9:2281-9.

65. Rahman A, Roy S, Muraleedharan NN, Phukan AK. Effects of potassium chloride and potassium sulphate on the efficacy of insecticides against infestation by Helopeltis theivora (Heteroptera: Miridae) in tea plantations. Int J Trop Insect Sci. 2014;33(3):217-21.

66. Deinlein U, Stephan AB, Horie T, Luo W, Xu G, Schroeder Jl. Plant salttolerance mechanisms. Trends Plant Sci. 2014;19(6):371-9.

67. Zhu JK. Abiotic stress signaling and responses in plants. Cell. 2016; 167(2):313-24.

68. Nelson BK, Cai X, Nebenfuhr A. A multicolored set of in vivo organelle markers for co-localization studies in Arabidopsis and other plants. Plant J. 2007:51(6):1126-36.

69. Wang Y, Wu W. Regulation of potassium transport and signaling in plants. Curr Opin Plant Biol. 2017;39:123-8.

70. Qin Y, Wu W, Wang Y. ZmHAK5 and ZmHAK1 function in $\mathrm{K}^{+}$uptake and distribution in maize under low $\mathrm{K}^{+}$conditions. J Integr Plant Biol. 2018;61:691-705

71. Okada T, Nakayama H, Shinmyo A, Yoshida K. Expression of OsHAK genes encoding potassium ion transporters in rice. Plant Biotechnol. 2008;25:241-5.

72. Rubio V, Bustos R, Irigoyen ML, Cardona-López X, Rojas-Triana M, Paz-Ares J. Plant hormones and nutrient signaling. Plant Mol Biol. 2008;69(4):361-73.

73. Munns M, Mark M. Mechanisms of salinity tolerance. Annu Rev Plant Biol. 2008;59:651-81.

74. Amtmann A, Hammond JP, Armengaud P, Patrick White PJ. Nutrient sensing and signalling in plants: potassium and phosphorus, vol. 43: Academic Press; 2005

75. Anschutz U, Becker D, Shabala S. Going beyond nutrition: regulation of potassium homoeostasis as a common denominator of plant adaptive responses to environment. J Plant Physiol. 2014;171(9):670-87.

76. Punta M, Coggill PC, Eberhardt RY, Mistry J, Tate J, Boursnell C, et al. The Pfam protein families database. Nucleic Acids Res. 2011;40(D1):D290-301.

77. Zdobnov EM, Apweiler R. InterProScan-an integration platform for the signature-recognition methods in InterPro. Bioinformatics. 2001;17(9):847-8.

78. Letunic I, Doerks T, Bork P. SMART 7: recent updates to the protein domain annotation resource. Nucleic Acids Res. 2012;40(D1):D302-5.

79. Tamura K, Stecher G, Peterson D, Filipski A, Kumar S. MEGA6: molecular evolutionary genetics analysis version 6.0. Mol Biol Evol. 2013;30(12):2725-9.

80. Shi C, Yang H, Wei C, Yu O, Zhang Z, Jiang C, Sun J, Li Y, Chen Q, Xia T, Wan $C$. Deep sequencing of the Camellia sinensis transcriptome revealed candidate genes for major metabolic pathways of tea-specific compounds. BMC Genomics. 2011;12:131.

81. Konishi S, Miyamoto S, Taki T. Stimulatory effects of aluminum on tea plants grown under low and high phosphorus supply. Soil Sci Plant Nutr. 1985;31(3):361-8.

82. Schmittgen TD, Zakrajsek BA. Effect of experimental treatment on housekeeping gene expression: validation by real-time, quantitative RT-PCR. J Biochem Biophys Methods. 2000;46:69-81.

83. Yang $T$, Huang $X$. Deep sequencing-based characterization of transcriptome of Pyrus ussuriensis in response to cold stress. Gene. 2018;661:109-18.

84. Liu J, Liu J, Liu J, Cui M, Huang Y, Tian Y, Chen A, Xu G. The potassium transporter SIHAK10 is involved in mycorrhizal potassium uptake. Plant Physiol. 2019;180(1):465-79.

85. Rubio F, Gassmann W, Schroeder Jl. Sodium-driven potassium uptake by the plant potassium transporter HKT1 and mutations conferring salt tolerance. Science. 1995;270(5242):1660-3.

\section{Publisher's Note}

Springer Nature remains neutral with regard to jurisdictional claims in published maps and institutional affiliations. 